\title{
A Monotonic Weighted Banzhaf Value for Voting Games
}

\author{
Conrado M. Manuel ${ }^{*}+$ (D) and Daniel Martín ${ }^{+}$(D) \\ Faculty of Statistics, Complutense University of Madrid, Puerta de Hierro, 1, 28040 Madrid, Spain; \\ daniem05@ucm.es \\ * Correspondence: conrado@estad.ucm.es \\ † These authors contributed equally to this work.
}

check for

updates

Citation: Manuel, C.M.; Martín, D. A Monotonic Weighted Banzhaf Value for Voting Games. Mathematics 2021, 9, 1343. https://doi.org/10.3390/ math9121343

Academic Editor: Vladimir Mazalov

Received: 21 April 2021

Accepted: 5 June 2021

Published: 10 June 2021

Publisher's Note: MDPI stays neutral with regard to jurisdictional claims in published maps and institutional affiliations.

Copyright: (c) 2021 by the authors. Licensee MDPI, Basel, Switzerland. This article is an open access article distributed under the terms and conditions of the Creative Commons Attribution (CC BY) license (https:// creativecommons.org/licenses/by/ $4.0 /)$.

\begin{abstract}
The aim of this paper is to extend the classical Banzhaf index of power to voting games in which players have weights representing different cooperation or bargaining abilities. The obtained value does not satisfy the classical total power property, which is justified by the imperfect cooperation. Nevertheless, it is monotonous in the weights. We also obtain three different characterizations of the value. Then we relate it to the Owen multilinear extension.
\end{abstract}

Keywords: game theory; cooperative game; Banzhaf value; weighted game; cooperation abilities

\section{Introduction}

A cooperative n-person game with transferable utility or a TU game is a model for the situations in which players can obtain benefits (measured by real numbers) when cooperating. In a TU game, it is assumed that all players are totally cooperative and that all coalitions are feasible. Formally, a TU game is a pair formed by a set of players and the characteristic function that assigns to each coalition (subset of players) a real number, which is the (transferable) utility that the members of that coalition can obtain by cooperating. Shapley [1] a introduced his well-known allocation rule or point solution for these situations. Another relevant point solution, introduced as a power index for voting games (monotonous games in which coalitions are winning or losing and thus their outcome is 1 or 0 , respectively), was suggested by Penrose [2], Banzhaf [3], and Coleman [4], and later generalized and characterized in the space of all TU games by Owen [5,6]. Game-theorists have paid a lot of attention to the Banzhaf value since it was introduced. Different characterizations of the Banzhaf value are given by Roth [7], Dubey and Shapley [8], Lehrer [9], Feltkamp [10], Dragan [11], Nowak [12], Laruelle and Valenciano [13], and Casajus [14], to name a few.

The Banzhaf value has also been defined for games with a priori unions obtaining the so-called Banzhaf-Owen value. It was introduced by Owen [15], and studied by AlonsoMeijide et al. [16] and Lorenzo-Freire [17]. Algaba et al. [18] extended and characterized the Banzhaf value to cooperative games on antimatroids, van den Brink [19,20] to games with a permission structure, Alonso-Meijide and Fiestras-Janeiro [21] to communication situations, Gallego et al. [22] to games with fuzzy communication structure, Tan et al. [23] to cooperative games with interval payoffs, and Fragnelli and Pusillo [24] applied the Banzhaf value to a multicriteria game to a microarray game.

The models in game theory are classically divided into cooperative and non-cooperative. Shapley [25] related cooperative and non-cooperative games by assuming that a TU game may have imperfect cooperation, that is, players can have different cooperation levels. He modeled this situation by associating a positive weight to each player. Then, he introduced the weighted Shapley value extending his rule to TU games [1]. The weighted Shapley value was generalized by Kalai and Samet [26].

The first attempt to obtain and characterize a weighted Banzhaf value was done by Radzik et al. [27] who analyzed cooperative games in which some a priori importance 
measures were assigned to the players. These measures are called weights. Mathematically, these are modeled as an exogenous vector of positive real numbers. The obtained solution is strongly inspired by the ideas used to define the weighted Shapley value and admits, as criticism, the lack of monotonicity in the weights (which is anti-intuitive). Precisely avoiding this weakness is at the origin of the present work: obtaining a weighted Banzhaf value that satisfies monotonicity in the weights.

Manuel and Martín [28] analyzed 'cooperative games in which players have different cooperation levels, different bargaining abilities or they are willing to make a different effort in cooperation'. In this case, the cooperation abilities are modeled by means of an n-vector of weights in $[0,1]$. The weights are assumed exogenous, and modulate the level, interest, or skill of the players in the cooperation or the bargaining. For example, unit weight players are fully cooperative as a typical player in a TU game, while a zero weight corresponds to players who are not interested at all in cooperating. Values of the weights between the two extremes rank the desire for cooperation. We assume the obvious difficulty of estimating these weights in practical situations. In our disclaimer, we will say that this difficulty is also associated with other weighted values.

Aubin [29] called a vector of weights in [0,1] a fuzzy coalition, in which components are regarded as the rate of participation of players in the fuzzy coalition. The parallelism between fuzzy cooperative games and our approach ends here, since the modification of the original characteristic function that we will introduce and the characteristic function of a fuzzy game are not related at all. A (zero-normalized) game with players having different cooperation abilities can also be described by a fuzzy communication structure complete by links [30,31]. Manuel and Martín [28] defined a weighted Shapley value which, unlike that obtained in [25], is not efficient (a consequence of the imperfect cooperation) but is monotonous in the weights, a weakness of the weighted Shapley value pointed out in Owen [32].

In this paper, we generalize the Banzhaf value to the setting of voting games with players having different cooperation abilities. These skills are very important in voting games, since the mere arithmetic of the distribution of sites is generally not sufficient to guarantee neither the formation nor the stability of coalitions. As an example, in Spain, after the election in 20 December 2015, among other causes, the lack of cooperation ability of parties motivated a new election in 26 June 2016, and after the election, in 28 April 2019, the lack of agreement to form a majority led to a new call for elections in 10 November 2019. The habitual institutional blockade of the Italian society is proverbial, which has undergone 66 governments since the proclamation of the republic in 1946, of which only six have lasted for more than two years.

As in [28], we will assume that, in these situations, the original voting game has to be modified by assuming that coalitions can only retain a fraction of their dividend (the minimum of their cooperation abilities, as it is not possible to obtain more cooperation than that one corresponding to the most compromising of the members) as a consequence of the imperfect cooperation. In our proposal, which is the Banzhaf value of the modified game, the imperfect cooperation reduces the total power of the grand coalition, but the obtained value is monotonous in the weights. As mentioned, this property is not satisfied by the weighted Banzhaf value of Radzik et al. [27]. Nevertheless, the total power holds for it. Moreover, we obtain several characterizations of the introduced value, some of them extending the more prominent ones existing in the literature for the (classical) Banzhaf value. The axioms used in the different characterizations refer to an assumed weight system and are, to some extent, parallel to the standard axioms used in other characterizations of the Banzhaf value. A different approach may be possible, in the spirit of Kalai and Samet [26], to provide axioms that allow to characterize a solution (such as the one proposed in this article) if, and only if a system of weights exists. We also obtain the relation between the multilinear extension of Owen $[5,33]$ and the defined value.

The remainder of the paper is organized as follows. After this introduction, there is a section of preliminaries. Section 3 is devoted to the definition of voting games with players 
having different cooperative abilities, as well as the definition of the modified game. We also analyze its properties. Then, in Section 4 the new value is introduced. Three different characterizations of it are included in Section 5. Section 6 is devoted to the relationship between multilinear extensions and the defined value. The article ends with a short section of final remarks and an extensive bibliography.

\section{Preliminaries}

A cooperative game with transferable utility (a TU game) is a pair $(N, v)$ in which $N=\{1, \ldots, n\}$ is the set of players and $v$ is the characteristic function defined on $2^{N}$. For each $S \subseteq N, v(S) \in \mathbb{R}$ is the outcome that the $s$ members of $S$ can obtain if they decide to cooperate. It is supposed that $v(\varnothing)=0$.

The family of the unanimity games $\left\{\left(N, u_{S}\right)\right\} \varnothing \neq S \subseteq N$ with $u_{S}$ given by:

$$
u_{S}(T)=\left\{\begin{array}{l}
1, \quad \text { if } S \subseteq T \\
0, \quad \text { otherwise }
\end{array}\right.
$$

is a basis of $G^{N}$, the vector space of all TU games with players set $N$. Then, for each $(N, v) \in G^{N}$,

$$
v=\sum_{\varnothing \neq S \subseteq N} \Delta_{v}(S) u_{S} .
$$

The coefficients $\Delta_{v}(S)$, for $S \subseteq N$, are known as Harsanyi dividends [34]. They can be written in terms of the value of the coalitions in the following way:

$$
\Delta_{v}(S)=\sum_{T \subseteq S}(-1)^{S-t} v(T), \text { for each } \varnothing \neq S \subseteq N .
$$

The worth of each coalition can also be calculated from Harsanyi dividends as:

$$
v(S)=\sum_{\varnothing \neq T \subseteq S} \Delta_{v}(T), \text { for each } \varnothing \neq S \subseteq N .
$$

A game $(N, v) \in G^{N}$ is (i) superadditive if, for all $S, T \subseteq N$ with $S \cap T=\varnothing, v(S \cup T) \geq$ $v(S)+v(T)$; (ii) convex, if for all $S, T \subseteq N, v(S \cup T)+v(S \cap T) \geq v(S)+v(T)$; (iii) zeronormalized, if $v(\{i\})=0$ for all $i \in N$. (iv) symmetric, if $v(S)=v(T)$ for $S, T \subseteq N$ with $s=t$; (v) monotonic, if $S \subseteq T \subseteq N$ implies $v(S) \leq v(T) \leq v(N)$ and (vi) simple, if for all $S \subseteq N, v(S) \in\{0,1\}$.

Given $(N, v) \in G^{N}$ and $S \subseteq N$, the restriction of $(N, v)$ to $S$ is the n-person game $\left(N, v_{\mid S}\right)$ with characteristic function $v_{\mid S}(T)=v(T \cap S)$ for all $T \subseteq N$.

A map $\psi: G^{N} \rightarrow \mathbb{R}^{n}$, with $\psi_{i}(N, v)$ representing the outcome for player $i$ in game $(N, v)$, is an allocation rule (point solution) for TU games.

Banzhaf [3] introduced a point solution in the domain of simple games with players set $N, S G^{N}$, which was mathematically developed later by Dubey and Shapley [8]. They defined a swing or swing for player $i$ as a pair of sets of the form $(S, S \backslash\{i\})$ such that $v(S \cup\{i\})-v(S)=1$. They noted $\eta_{i}(v)$ for the number of swings of player $i$ and $\bar{\eta}(v)$ for the total number of swings in the game $v \in S G^{N}$. They named $\eta_{i}$ as the 'raw' Banzhaf index for player $i$, and characterized it using the following four axioms:

(i) For all $(N, v) \in S G^{N}, \sum_{i \in N} \psi_{i}(N, v)=\bar{\eta}(v)$.

(ii) Null player property: for all $(N, v) \in S G^{N}$ and $i \in N$ such that $\eta_{i}=0, \psi_{i}(N, v)=0$.

(iii) Anonymity: for any permutation $\pi$ of $N, \psi_{\pi(i)}(N, \pi v)=\psi_{i}(N, v)$. For $(N, v) \in S G^{N}$ the characteristic function $\pi v$ is defined as $(\pi v)(S)=v\left(\pi^{-1}(S)\right)$, for all $S \subseteq N$.

(iv) Transfer property: for any $(N, v),(N, w) \in S G^{N}, \psi(N, v \vee w)+\psi(N, v \wedge w)=\psi(N, v)+$ $\psi(N, w)$. For $(N, v),(N, w) \in S G^{N}$, the characteristic functions $v \vee w$ and $v \wedge w$ are defined as: $(v \vee w)(S)=\max \{v(S), w(S)\}$ and $(v \wedge w)(S)=\min \{v(S), w(S)\}$, for all $S \subseteq N$. 
The normalization of the previous index to obtain the proportion of power has been a common practice. The most frequent normalizations are $\frac{\eta_{i}(v)}{\bar{\eta}(v)}$ and $\frac{\eta_{i}(v)}{2^{n-1}}$, for $i=1, \ldots, n$.

Owen [5] extended the (normalized, dividing by $2^{n-1}$ ) Banzhaf index to $G^{N}$. Thus, for $(N, v) \in G^{N}$,

$$
B_{i}(N, v)=\sum_{S \subseteq N \backslash\{i\}} \frac{1}{2^{n-1}}[v(S \cup\{i\})-v(S)] .
$$

He used the multilinear extension of a game [33] to calculate it [5], applied this value to the presidential election game [6], and characterized it $[5,6]$.

The Banzhaf value can also be expressed in terms of the dividends, as:

$$
B_{i}(N, v)=\sum_{i \in S \subseteq N} \frac{\Delta_{v}(S)}{2^{s-1}}, i \in N .
$$

\section{Voting Games with Players Having Different Cooperation Abilities 3.1. Voting Games}

The idea of decision-making by voting is formalized in the following definition.

Definition 1. The game $(N, v) \in G^{N}$ is a voting game if

(i) $v(S) \in\{0,1\}$ for all $S \subseteq N$.

(ii) $v(N)=1$.

(iii) If $S, T \in 2^{N}$ with $S \subseteq T$, then $v(S) \leq v(T)$.

$S$ is a winner coalition in the voting game $(N, v)$ if $v(S)=1$, and it is a losing coalition otherwise. Then, a voting game the grand coalition is necessarily a winning one, and supercoalitions of a winning coalition are also winning coalitions.

A voting game is superadditive (or proper) if, for every $S, T \subseteq N$, with $S \cap T=\varnothing$, then $v(S \cup T) \geq v(S)+v(T)$ holds. As a consequence, in a superadditive voting game two winning coalitions cannot be disjoint; that is, if $S, T \in 2^{N}$, with $v(S)=v(T)=1$, then $S \cap T \neq \varnothing$.

A player $i \in N$ is a dictator in the proper voting game $(N, v)$, if $v(\{i\})=1$. We can have only one dictator in a proper voting game. In the following, we will only consider proper voting games with no dictator. Nevertheless, by misuse of the language, sometimes we will use voting game for a proper voting game with no dictator. $G_{p}^{N}$ will denote the family of these voting games.

A voting game $(N, v) \in G_{p}^{N}$ is convex if, for all $S, T \in 2^{N}, v(S \cup T)+v(S \cap T) \geq$ $v(S)+v(T)$ holds. In a convex voting game, the winning coalitions cannot be disjoint (as in proper voting games) and moreover, their intersection has to also be a winning coalition.

$S$ is a minimal winning coalition in $(N, v) \in G_{p}^{N}$ if $v(S)=1$, and for all $T \subsetneq S$, $v(T)=0$.

Proposition 1. Ref. [35] If $(N, v)$ is a voting game and $\mathcal{M W C}(N, v)=\left\{S_{1}, S_{2}, \ldots, S_{r}\right\}$ is the family of its minimal winning coalitions, we have $v=u_{S_{1}} \vee \cdots \vee u_{S_{r}}=\bigvee_{i=1}^{r} u_{S_{i}}$.

Proposition 2. A convex voting game is necessarily a unanimity game.

Proof. It is clear that in a voting game there is at least one minimal winning coalition. Suppose $(N, v)$ is a convex voting game and $S_{1}, S_{2}, S_{1} \neq S_{2}$ are both minimal winning coalitions in it. Then, as $(N, v)$ is convex, and $S_{1} \cap S_{2}$ must be a winning coalition. Nevertheless, $S_{1} \cap S_{2} \subsetneq S_{1}$ and $S_{1} \cap S_{2} \subsetneq S_{2}$, which contradicts that $S_{1}$ and $S_{2}$ are minimal. Then, the game $(N, v)$ can have only one minimal winning coalition $S$, and thus, using the previous proposition, $v=u_{S}$. 
In the following proposition, we obtain an alternative expression for a proper voting game, which allows us to know (in the next corollary) its dividends.

Proposition 3. If $(N, v) \in G_{p}^{N}$ and $\mathcal{M W C}(N, v)=\left\{S_{1}, S_{2}, \ldots, S_{r}\right\}$, then:

$$
v=\mathbf{1}-\prod_{i=1}^{r}\left(\mathbf{1}-u_{S_{i}}\right) .
$$

We denote by $(N, \mathbf{1})$ the game in which $v(S)=1$ for all $S \subseteq N, S \neq \varnothing$. That is, the voting game in which all non-empty coalitions are winning. Of course, $(N, \mathbf{1})$ is not a proper voting game, and thus the previous formula must be understood as an expression of $(N, v)$ in $G^{N}$.

Proof. Suppose $\varnothing \neq S \subseteq N$ is a winning coalition in $(N, v)$, that is, $v(S)=1$. Then, there is $l \in\{1,2, \ldots, r\}$ such that $S_{l} \subseteq S$, and thus $u_{S_{l}}(S)=1$. As a consequence, $\left[\mathbf{1}-\prod_{i=1}^{r}\left(\mathbf{1}-u_{S_{i}}\right)\right](S)=\mathbf{1}(S)=1$.

On the other hand, if $S$ is a losing coalition, $u_{S_{i}}(S)=0$ for $i=1, \ldots, r$, as $S$ does not contain any minimal winning coalition (in such a case, $S$ is also a winning coalition). Then, $0=v(S)=\left[\mathbf{1}-\prod_{i=1}^{r}\left(\mathbf{1}-u_{S_{i}}\right)\right](S)$, and thus the result is proved.

Corollary 1. If $(N, v) \in G_{p}^{N}$ is a voting game and $\mathcal{M W C}(N, v)=\left\{S_{1}, S_{2} \ldots S_{r}\right\}$, then

$$
v=\sum_{i=1}^{r} u_{S_{i}}-\sum_{i=1}^{r-1} \sum_{j=i+1}^{r} u_{S_{i} \cup S_{j}}+\sum_{i=1}^{r-2} \sum_{j=i+1}^{r-1} \sum_{k=j+1}^{r} u_{S_{i} \cup S_{j} \cup S_{k}}+\cdots+(-1)^{r-1} u_{S_{1} \cup S_{2} \cup \cdots \cup S_{r}} .
$$

Proof. It is a direct consequence of the previous proposition that for $i, j \in\{1, \ldots, r\}$, $u_{S_{i}} \cdot u_{S_{j}}=u_{S_{i} \cup S_{j}}$.

Remark 1. If a proper voting game $(N, v)$ has a dictator (Player 1 , for example), then $v=u_{\{1\}}$. The simplicity of these situations motivated us to ignore them.

\subsection{The Modified Game}

As mentioned previously, Shapley [25] extended the classical definition of a TU game to include the possibility that players can have different cooperation or bargaining abilities. He also defined a solution, known as the weighted Shapley value, for these situations. With the aim of introducing a weighted Banzhaf value, Radzik et al. [27] also assumed that the players had some measures of importance or weights. In both contributions, an exogenous weight in $\mathbb{R}^{+}$is assigned to each player. Manuel and Martín [28] assigned a vector of parameters $\lambda=\left(\lambda_{1}, \ldots, \lambda_{n}\right) \in[0,1]^{n}$ to the players in a TU game $(N, v)$. These parameters measure the level of cooperation of the players as in [25], and they can also be seen as a fuzzy coalition [29]. Then, they modified the original game to a new one $\left(N, v^{\lambda}\right)$ with a characteristic function:

$$
v^{\lambda}(S)=\sum_{T \subseteq S, t \geq 2} \Delta_{v}(T) \min _{i \in T}\left\{\lambda_{i}\right\}+\sum_{i \in S} \Delta_{v}(\{i\}),
$$

for all $S \subseteq N, S \neq \varnothing$. They propose the Shapley value [1] of this modified game as a monotonous weighted Shapley value.

Next, we restrict the definition of [28] to voting games.

Definition 2. A voting game with players having different cooperation abilities is a triple $(N, v, \lambda)$, in which $(N, v) \in G_{p}^{N}$ and $\lambda=\left(\lambda_{1}, \ldots, \lambda_{n}\right)$ is a fixed vector in $[0,1]^{n} . \lambda_{i}$ represents the 
cooperation ability of $i \in N . G_{p, \Lambda}^{N}$ represents the set of all proper voting games with players having different bargaining abilities.

Remark 2. We will identify $G_{p}^{N}$ with the family of $(N, v, \lambda) \in G_{p, \Lambda}^{N}$ in which $\lambda=(1, \ldots, 1)$.

Remark 3. As for $(N, v, \lambda) \in G_{p, \Lambda}^{N}$, the underlying game is zero-normalized, and $(N, v, \lambda)$ can be described by a fuzzy communication structure complete by links [30,31].

Regarding [28], we can associate to each voting game with players having different bargaining abilities the modified game in which players in a unanimity game can only retain a fraction of the total unit payoff. In the definition of [28], it is supposed that each singleton coalition can retain its dividend as each player is fully cooperative with himself. In our framework, as dictators are out of the question, we do not need to consider dividends of singletons. In any case, if a dictator is taken into account, of course his dividend must be unchanged.

The underlying idea is that the maximum level of cooperation in a unanimity game must coincide with the corresponding to the less cooperative player. Then we have the following definition:

Definition 3. Given $(N, v, \lambda) \in G_{p, \Lambda^{\prime}}^{N}$ the modified game $\left(N, v^{\lambda}\right) \in G^{N}$ has a characteristic function given by

$$
\begin{gathered}
v^{\lambda}=\sum_{i=1}^{r} \min _{k \in S_{i}}\left\{\lambda_{k}\right\} u_{S_{i}}-\sum_{i=1}^{r-1} \sum_{j=i+1}^{r} \min _{k \in S_{i} \cup S_{j}}\left\{\lambda_{k}\right\} u_{S_{i} \cup S_{j}} \\
+\cdots+(-1)^{r-1} \min _{k \in S_{1} \cup S_{2} \cup \cdots \cup S_{r}}\left\{\lambda_{k}\right\} u_{S_{1} \cup S_{2} \cup \cdots \cup S_{r}}
\end{gathered}
$$

, where $\mathcal{M W C}(N, v)=\left\{S_{1}, S_{2}, \ldots, S_{r}\right\}$

Remark 4. It is clear that the modified game will not be, in general, a voting game as $v^{\lambda}(N)$ can be smaller than one.

Let us consider the Example 3.1 in [28].

Example 1. In a given parliament, the distribution of seats is $16 \%, 35 \%, 9 \%$, and $40 \%$, respectively, for parties 1,2,3, 4 which are aligned in a left-right spectrum. To pass a bill, more than $50 \%$ of votes are needed. Then, the minimal winning coalitions of this voting game $(N, v)$ are $\{1,2\},\{1,4\}$ and $\{2,4\}$. Therefore,

$$
\begin{gathered}
v=u_{\{1,2\}}+u_{\{1,4\}}+u_{\{2,4\}}-u_{\{1,2,4\}}-u_{\{1,2,4\}}-u_{\{1,2,4\}}+u_{\{1,2,4\}} \\
=u_{\{1,2\}}+u_{\{1,4\}}+u_{\{2,4\}}-2 u_{\{1,2,4\}} .
\end{gathered}
$$

If $\lambda=(0.2,1,0.8,0.1)$, the characteristic function of $\left(N, v^{\lambda}\right)$ is given by

$$
v^{\lambda}=0.2 u_{\{1,2\}}+0.1 u_{\{1,4\}}+0.1 u_{\{2,4\}}-0.2 u_{\{1,2,4\}} .
$$

In the following proposition, we obtain an alternative expression for $v^{\lambda}(S)$ when $(N, v)$ is a voting game.

Proposition 4. Consider $(N, v, \lambda) \in G_{p, \Lambda^{\prime}}^{N}$ and $S \subseteq N$. Then,

$$
v^{\lambda}(S)=\max _{j=1, \ldots, l} \min _{k \in S_{i_{j}}}\left\{\lambda_{k}\right\}
$$


if $\mathcal{M W C}(S, v)=\left\{S_{i_{1}}, \ldots, S_{i_{l}}\right\} \neq \varnothing$ is the family of the minimal winning coalitions of $(N, v)$ contained in $S$, and

$$
v^{\lambda}(S)=0, \text { if } \mathcal{M W C}(S, v)=\varnothing .
$$

Proof. Suppose that $\mathcal{M W C}(N, v)=\left\{S_{1}, S_{2}, \ldots, S_{r}\right\}$. For $S \subseteq N$, let us denote $\lambda_{S}=$ $\min _{k \in S}\left\{\lambda_{k}\right\}$. Then,

$$
v^{\lambda}=\sum_{i=1}^{r} \lambda_{S_{i}} u_{S_{i}}-\sum_{i=1}^{r-1} \sum_{j=i+1}^{r} \lambda_{S_{i} \cup S_{j}} u_{S_{i} \cup S_{j}}+\cdots+(-1)^{r-1} \lambda_{S_{1} \cup \ldots \cup S_{r}} u_{S_{1} \cup \cdots \cup S_{r}},
$$

and thus, it is straightforward that $v^{\lambda}(S)=0$ if $S_{i} \not \subseteq S$ for $i=1, \ldots, r$.

Consider $S \subseteq N$, and suppose that $\mathcal{M W C}(S, v)=\left\{S_{i_{1}}, \ldots, S_{i_{l}}\right\} \subseteq \mathcal{M W C}(N, v)$ is the set of the minimal winning coalitions of $(N, v)$ that are contained in $S$. Without loss of generality, let us assume that $\lambda_{S_{i_{1}}} \leq \lambda_{S_{i_{2}}} \leq \cdots \leq \lambda_{S_{i_{l}}}$. Then,

$$
\begin{gathered}
v^{\lambda}(S)=\left[\sum_{i=1}^{r} \lambda_{S_{i}} u_{S_{i}}-\sum_{i=1}^{r-1} \sum_{k=i+1}^{r} \min \left\{\lambda_{S_{i}}, \lambda_{S_{k}}\right\} u_{S_{i} \cup S_{k}}\right. \\
\left.+\cdots+(-1)^{r-1} \min \left\{\lambda_{S_{1}}, \ldots, \lambda_{S_{r}}\right\} u_{S_{1} \cup \cdots \cup S_{r}}\right](S) \\
=\left[\sum_{j=1}^{l} \lambda_{S_{i_{j}}} u_{S_{i_{j}}}-\sum_{j=1}^{l-1} \sum_{k=j+1}^{l} \min \left\{\lambda_{S_{i_{j}}}, \lambda_{S_{i_{k}}}\right\} u_{S_{i_{j}} \cup S_{i_{k}}}\right. \\
\left.+\cdots+(-1)^{l-1} \min \left\{\lambda_{S_{i_{1}}}, \ldots, \lambda_{S_{i_{l}}}\right\} u_{S_{i_{1}} \cup \ldots \cup S_{i_{l}}}\right](S) \\
=\left[\sum_{j=1}^{l} \lambda_{S_{i_{j}}} u_{S_{i_{j}}}-\sum_{j=1}^{l-1} \sum_{k=j+1}^{l} \lambda_{S_{i_{j}}} u_{S_{i_{j}} \cup S_{i_{k}}}+\cdots+(-1)^{l-1} \lambda_{S_{i_{1}}} u_{S_{i_{1}} \cup \ldots \cup S_{i_{l}}}\right](S),
\end{gathered}
$$

with the second equality holding as, for $i \neq i_{1}, \ldots, i_{l}, S_{i} \nsubseteq \subseteq$, and the last one because $\lambda_{S_{i_{1}}} \leq \lambda_{S_{i_{2}}} \leq \cdots \leq \lambda_{S_{i_{l}}}$.

Then,

$$
\begin{gathered}
v^{\lambda}(S)=\lambda_{S_{i_{1}}}\left[1-\left(\begin{array}{c}
l-1 \\
1
\end{array}\right)+\left(\begin{array}{c}
l-1 \\
2
\end{array}\right)+\cdots+(-1)^{l-1}\left(\begin{array}{l}
l-1 \\
l-1
\end{array}\right)\right] \\
+\lambda_{S_{i_{2}}}\left[1-\left(\begin{array}{c}
l-2 \\
1
\end{array}\right)+\cdots+(-1)^{l-2}\left(\begin{array}{l}
l-2 \\
l-2
\end{array}\right)\right] \\
+\cdots+\lambda_{S_{i_{l-1}}}\left[1-\left(\begin{array}{l}
1 \\
1
\end{array}\right)\right]+\lambda_{S_{i_{l}}}=\lambda_{S_{i_{l}}}
\end{gathered}
$$

where we have needed the binomial identities

$$
1-\left(\begin{array}{l}
t \\
1
\end{array}\right)+\left(\begin{array}{l}
t \\
2
\end{array}\right)+\cdots+(-1)^{t}\left(\begin{array}{l}
t \\
t
\end{array}\right)=(1-1)^{t}=0, \text { for } t \in N, t \geq 1
$$

Thus,

$$
v^{\lambda}(S)=\lambda_{S_{i_{l}}}=\max \left\{\lambda_{S_{i_{1}}}, \lambda_{S_{i_{2}}}, \ldots, \lambda_{S_{i_{l}}}\right\}=\max _{j=1, \ldots, l} \min _{k \in S_{i_{j}}}\left\{\lambda_{k}\right\},
$$

which proves the result.

Example 2. Consider again the voting game in which $N=\{1,2,3,4\}$,

$$
v=u_{\{1,2\}}+u_{\{1,4\}}+u_{\{2,4\}}-2 u_{\{1,2,4\}},
$$

and the bargaining abilities are given by $\lambda=(0.2,1,0.8,0.1)$. 
Then, for $S=\{1,3\}, v^{\lambda}(S)=0$, as none of the minimal winning coalitions $\{1,2\},\{1,4\}$, $\{2,4\}$ is contained in $S$.

For $S=\{1,2,4\}, \mathcal{M W C}(S, v)=\{\{1,2\},\{1,4\},\{2,4\}\}$, and thus,

$$
v^{\lambda}(S)=\left[0.2 u_{\{1,2\}}+0.1 u_{\{1,4\}}+0.1 u_{\{2,4\}}-0.2 u_{\{1,2,4\}}\right](S)=0.2 .
$$

On the other hand, from Proposition 4,

$$
\begin{gathered}
v^{\lambda}(S)=\max \left\{\lambda_{\{1,2\}}, \lambda_{\{1,4\}}, \lambda_{\{2,4\}}\right\}=\max \left\{\min \left\{\lambda_{1}, \lambda_{2}\right\}, \min \left\{\lambda_{1}, \lambda_{4}\right\}, \min \left\{\lambda_{2}, \lambda_{4}\right\}\right\} \\
=\max \{0.2,0.2,0.1\}=0.2 .
\end{gathered}
$$

In the following proposition we prove that the modified voting game can be written as a positive lineal combination of voting games. In particular, the obtained expression can be seen as the Choquet extension of the original voting game [36-39].

Proposition 5. Given $(N, v, \lambda) \in G_{p, \Lambda^{\prime}}^{N}$ suppose $\operatorname{MWC}(N, v)=\left\{S_{1}, \ldots, S_{r}\right\}$ is the family of the minimal winning coalitions of $(N, v)$. If $\lambda_{S_{i}}=\min _{j \in S_{i}}\left\{\lambda_{j}\right\}$ for $j=1,2, \ldots, r$, with $\lambda_{S_{1}} \leq \lambda_{S_{2}} \leq$ $\cdots \leq \lambda_{S_{r}}$, and we define $\lambda_{S_{0}}=0$, then,

$$
v^{\lambda}=\sum_{h=0}^{r-1}\left(\lambda_{S_{h+1}}-\lambda_{S_{h}}\right) \cdot v_{\left.\right|_{S_{h+1} \cup \cdots \cup S_{r}}}
$$

Proof. From the previous proposition, we have that, for $S \subseteq N$,

$$
v^{\lambda}(S)=\max _{j=1, \ldots, l, l} \min _{k \in S_{i_{j}}}\left\{\lambda_{k}\right\}
$$

with $\varnothing \neq\left\{S_{i_{1}}, \ldots, S_{i_{l}}\right\} \subseteq \mathcal{M W C}(N, v)$ the set of minimal winning coalitions of $(N, v)$ are subsets of $S$, and $v^{\lambda}(S)=0$ if each element of $\mathcal{M W C}(N, v)$ is not contained in $S$.

For $h=0,1,2, \ldots, r, v_{\left.\right|_{S_{h+1}} \cup \cdots \cup S_{r}}$ is a linear combination of unanimity games of $S_{h+1}, \ldots, S_{r}$ and of their unions. Then, both members in (1) clearly give zero when applied to $S$ with $v^{\lambda}(S)=0$.

On the other hand, for $S \subseteq N$ such that

$$
v^{\lambda}(S)=\max _{j=1, \ldots, l} \min _{k \in S_{i_{j}}}\left\{\lambda_{k}\right\}=\lambda_{S_{i_{l}}}
$$

using again the expression of $v_{\left.\right|_{S_{h+1}} \cup \ldots \cup S_{r}}$ for $h=0,1, \ldots, r-1$, in terms of the unanimity games, and the fact that $S_{i_{l}+1}, \ldots, S_{r}$ are the elements in $\mathcal{M W C}(N, v)$ not contained in $S$, we have

$$
v^{\lambda}(S)=\sum_{h=0}^{r-1}\left(\lambda_{S_{h+1}}-\lambda_{S_{h}}\right) \cdot v_{\left.\right|_{S_{h+1}} \cup \cdots \cup S_{r}}(S)=\sum_{h=0}^{i_{l}-1}\left(\lambda_{S_{h+1}}-\lambda_{S_{h}}\right)=\lambda_{S_{i_{l}}},
$$

which completes the proof.

Example 3. Consider again the voting game in which $N=\{1,2,3,4\}$,

$$
v=u_{\{1,2\}}+u_{\{1,4\}}+u_{\{2,4\}}-2 u_{\{1,2,4\}}
$$

and the players have cooperation abilities given by $\lambda=(0.2,1,0.8,0.1)$. In the Example 2 , we obtained that

$$
v^{\lambda}=0.2 u_{\{1,2\}}+0.1 u_{\{1,4\}}+0.1 u_{\{2,4\}}-0.2 u_{\{1,2,4\}} .
$$


We have that $\mathcal{M W C}(N, v)=\left\{S_{1}=\{1,4\}, S_{2}=\{2,4\}, S_{3}=\{1,2\}\right\}$ and $\lambda_{S_{1}}=0.1, \lambda_{S_{2}}=0.1$ and $\lambda_{S_{3}}=0.2$ and thus, using the previous proposition,

$$
\begin{gathered}
\lambda_{S_{1}} v_{S_{1} \cup S_{2} \cup S_{3}}+\left(\lambda_{S_{2}}-\lambda_{S_{1}}\right) v_{\left.\right|_{S_{2} \cup S_{3}}}+\left(\lambda_{S_{3}}-\lambda_{S_{2}}\right) v_{\left.\right|_{3}} \\
=0.1 v_{\left.\right|_{1} \cup S_{2} \cup S_{3}}+0.1 v_{\left.\right|_{S_{3}}}=0.1\left(u_{\{1,2\}}+u_{\{1,4\}}+u_{\{2,4\}}-2 u_{\{1,2,4\}}\right)+0.1 u_{\{1,2\}} \\
=0.2 u_{\{1,2\}}+0.1 u_{\{1,4\}}+0.1 u_{\{2,4\}}-0.2 u_{\{1,2,4\}}=v^{\lambda}
\end{gathered}
$$

Remark 5. Essentially, this expression (1) coincides with the Choquet one for games with players having different bargaining abilities that is obtained in [28]. In that paper, for each $(N, v, \lambda)$ the authors define $y_{h}$, for $h=0,1, \ldots, r$, where $r \leq n$ is the number of different values among the weights of players in $(N, v, \lambda)$, in the following way: $y_{0}=0$, and for $h=1,2, \ldots, r, y_{h}=$ $\min _{i \in N}\left\{\lambda_{i} \mid \lambda_{i}>y_{h-1}\right\}$. Then, they prove that

$$
v^{\lambda}=\sum_{h=0}^{r-1}\left(y_{h+1}-y_{h}\right) v_{\mid N_{h+1}}
$$

with $N_{h+1}=\left\{i \in N \mid \lambda_{i} \geq y_{h+1}\right\}$, for $h=0, \ldots, r-1$. Applying this expression in the previous example, we have that $y_{1}=0.1, y_{2}=0.2, y_{3}=0.8, y_{4}=1, N_{1}=N, N_{2}=N \backslash\{4\}$, $N_{3}=N \backslash\{1,4\}$ and $N_{4}=N \backslash\{1,3,4\}$. Then,

$$
\begin{aligned}
& v^{\lambda}=y_{1} v+\left(y_{2}-y_{1}\right) v_{\left.\right|_{N \backslash\{4\}}}+\left(y_{3}-y_{2}\right) v_{\left.\right|_{N \backslash\{1,4\}}}+\left(y_{4}-y_{3}\right) v_{\left.\right|_{N \backslash\{1,3,4\}}} \\
& =0.1 v+(0.2-0.1) v_{\left.\right|_{N \backslash\{4\}}}+(0.8-0.2) v_{\left.\right|_{N \backslash\{1,4\}}}+(1-0.8) v_{\left.\right|_{N \backslash\{1,3,4\}}} .
\end{aligned}
$$

As $v_{\left.\right|_{N \backslash\{1,4\}}}=0$ and $v_{\left.\right|_{N \backslash\{1,3,4\}}}=0$, we obtain the expression in (2).

\subsection{Properties of the Modified Game}

In the following proposition, we summarize some properties of the modified game. First of all, from Remark 3 in [28], we have that if $\lambda=\mathbf{1}$, that is, if all players fully cooperate, the modified game $v^{\lambda}$ coincides with the original game $v$; if all players have an equal level of cooperation, the modified game is strategically equivalent to the original one, and finally, if $\lambda=\mathbf{0}$, then $v^{\lambda}=0$, that is, if players do not cooperate at all, the modified game becomes inessential.

Proposition 6. Given $(N, v, \lambda) \in G_{p, \Lambda}^{N}$ we have:

(i) If $\lambda$ is such that $\lambda_{i}>0$ for some $i \in N$, and $\lambda_{j}=0$ for $j \neq i$, then $v^{\lambda}$ is the null game. Thus, cooperation cannot emerge if only one player wants to cooperate.

(ii) If $\lambda_{i}=0$ for some $i \in N$, then $v^{\lambda}=\left(v_{\left.\right|_{N \backslash\{i\}}}\right)^{\lambda}=\left(v^{\lambda}\right)_{\left.\right|_{N \backslash\{i\}}}$. In particular, if $\lambda_{i}=0$ for some $i \in N$ and $\lambda_{j}=1$ for $j \neq i$, then $v^{\lambda}=v_{\left.\right|_{N \backslash\{i\}}}$. Thus, the lack of cooperation of a player is equivalent to the player leaving the game.

(iii) If $\lambda_{i}=0$ for some $i \in N$, then $v^{\lambda}=\left(v_{\mid s_{i_{1}} \cup \ldots \cup s_{i_{t}}}\right)^{\lambda}=\left(v^{\lambda}\right)_{\left.\right|_{i_{i_{1}} \cup \ldots \cup s_{i_{t}}}}$, where $S_{i_{1}}, \ldots, S_{i_{t}}$ are the minimal winning coalitions of $(N, v)$ such that $i \notin S_{i_{l}}, l=1, \ldots, t$. Thus, if a player is not willing to cooperate, all the minimal winning coalitions to which he belongs become loser coalitions.

(iv) If $\lambda, \lambda^{\prime}$ satisfy $\lambda_{i}^{\prime}>\lambda_{i}$ and $\lambda_{j}^{\prime}=\lambda_{j}$ for $j \neq i$ then,

$$
v^{\lambda^{\prime}}(S \cup\{i\})-v^{\lambda^{\prime}}(S) \geq v^{\lambda}(S \cup\{i\})-v^{\lambda}(S),
$$

for $S \subseteq N \backslash\{i\}$. Hence, when increasing the cooperation level of a player, his marginal contributions do not decrease. 
Proof. (i) From Proposition 4, given $S \subseteq N$, if $\mathcal{M W C}(S, v)=\left\{S_{i_{1}}, \ldots, S_{i_{l}}\right\} \neq \varnothing$ is the family of the minimal winning coalitions of $(N, v)$ contained in $S$ (recall that each minimal winning coalition has at least two players as the game has no dictator), we have

$$
v^{\lambda}(S)=\max _{j=1, \ldots, l} \min _{k \in S_{i_{j}}}\left\{\lambda_{k}\right\}=0
$$

and

$$
v^{\lambda}(S)=0, \text { if } \mathcal{M W C}(S, v)=\varnothing .
$$

(ii) Suppose $\mathcal{M W C}(N, v)=\left\{S_{1}, \ldots, S_{r}\right\}$ is the family of the minimal winning coalitions of $(N, v)$. If $\lambda_{i}=0$ for some $i \in N$, then $\min _{k \in T}\left\{\lambda_{k}\right\}=0$ for all $T \subseteq N$ such that $i \in T$. Thus, if $S_{i_{1}}, \ldots, S_{i_{t}}$ are the minimal winning coalitions of $(N, v)$ such that $i \notin S_{i_{l}}, l=1, \ldots, t$,

$$
\begin{gathered}
v^{\lambda}=\sum_{j=1}^{t} \min _{k \in S_{i_{j}}}\left\{\lambda_{k}\right\} u_{S_{i_{j}}}-\sum_{j=1}^{t-1} \sum_{l=j+1}^{t} \min _{k \in S_{i_{j}} \cup S_{i_{l}}}\left\{\lambda_{k}\right\} u_{S_{i_{j}} \cup S_{i_{l}}} \\
+\cdots+(-1)^{t-1} \min _{k \in S_{i_{1}} \cup S_{i_{2}} \cup \cdots \cup S_{i_{t}}}\left\{\lambda_{k}\right\} u_{S_{i_{1}} \cup S_{i_{2}} \cup \cdots \cup S_{i_{t}}}=\left(v_{\left.\right|_{N \backslash\{i\}}}\right)^{\lambda}=\left(v^{\lambda}\right)_{\left.\right|_{N \backslash\{i\}} .}
\end{gathered}
$$

In particular, if $\lambda_{j}=1$ for $j \neq i$, then $v_{\left.\right|_{N \backslash\{i\}} ^{\lambda}}^{\lambda}=v_{\left.\right|_{N \backslash\{i\}}}$.

(iii) It is straightforward from $i i$ ).

(iv) Consider $i \in N$ and $S \subseteq N \backslash\{i\}$. If $\mathcal{M W C}(S, v)=\left\{S_{i_{1}}, \ldots, S_{i_{l}}\right\}$, then

$$
v^{\lambda^{\prime}}(S)=\max _{j=1, \ldots, l} \min _{k \in S_{i_{j}}}\left\{\lambda_{k}^{\prime}\right\}=\max _{j=1, \ldots, l} \min _{k \in S_{i_{j}}}\left\{\lambda_{k}\right\}=v^{\lambda}(S),
$$

where the second equality holds as for $j=1, \ldots, l, i \notin S_{i_{j}}$ given that $S_{i_{j}} \subseteq S$. Then, for each player in $S_{i_{j}}, j=1, \ldots, l$, his weight is the same in $\lambda^{\prime}$ and $\lambda$.

Suppose that $\left\{S_{i_{1}}, \ldots, S_{i_{l}}, S_{i_{l+1}}, \ldots, S_{i_{h}}\right\}$ is the family of minimal winning coalitions of $(N, v)$ included in $S \cup\{i\}$. Then,

$$
v^{\lambda}(S \cup\{i\})=\max _{j=1, \ldots, h} \min _{k \in S_{i_{j}}}\left\{\lambda_{k}\right\} \leq \max _{j=1, \ldots, h, h \in \min _{i_{j}}}\left\{\lambda_{k}^{\prime}\right\}=v^{\lambda^{\prime}}(S \cup\{i\}),
$$

the inequality holding as $\lambda_{i}^{\prime} \geq \lambda_{i}$ and as $i$ can eventually be the player with the smaller weight in a minimal winning coalition. This completes the proof.

The following lemma precedes a proposition in which we prove that the modified game of a maximum of voting games is the maximum of its modified games.

Lemma 1. Let $\left(N, u_{S_{1}}, \lambda\right),\left(N, u_{S_{2}}, \lambda\right), \ldots,\left(N, u_{S_{r}}, \lambda\right) \in G_{p, \Lambda}^{N}$. Then,

$$
\left(u_{S_{1}} \vee u_{S_{2}} \vee \cdots \vee u_{S_{r}}\right)^{\lambda}=u_{S_{1}}^{\lambda} \vee u_{S_{2}}^{\lambda} \vee \cdots \vee u_{S_{r}}^{\lambda}
$$

Proof. Suppose $\lambda_{S_{i}}=\min _{k \in S_{i}}\left\{\lambda_{k}\right\}$ for $i=1, \ldots, r$, and, without loss of generality, let us assume that $\lambda_{S_{1}} \leq \lambda_{S_{2}} \leq \cdots \leq \lambda_{S_{r}}$. Then,

$$
\begin{gathered}
\left(u_{S_{1}} \vee u_{S_{2}} \vee \cdots \vee u_{S_{r}}\right)^{\lambda}=\left[\sum_{i=1}^{r} u_{S_{i}}-\sum_{i=1}^{r-1} \sum_{j=i+1}^{r} u_{S_{i} \cup S_{j}}+\sum_{i=1}^{r-2} \sum_{j=i+1}^{r-1} \sum_{l=j+1}^{r} u_{S_{i} \cup S_{j} \cup S_{l}}\right. \\
\left.+\ldots+(-1)^{r-1} u_{S_{1} \cup S_{2} \cup \ldots \cup S_{r}}\right]^{\lambda}=\sum_{i=1}^{r} \lambda_{S_{i}} u_{S_{i}}-\sum_{i=1}^{r-1} \sum_{j=i+1}^{r} \min _{k \in S_{i} \cup S_{j}}\left\{\lambda_{k}\right\} u_{S_{i} \cup S_{j}}
\end{gathered}
$$




$$
\begin{gathered}
+\sum_{i=1}^{r-2} \sum_{j=i+1}^{r-1} \sum_{l=j+1}^{r} \min _{k \in S_{i} \cup S_{j} \cup S_{l}}\left\{\lambda_{k}\right\} u_{S_{i} \cup S_{j} \cup S_{l}} \\
+\cdots+(-1)^{r-1} \min _{k \in S_{i} \cup S_{j} \cup \cdots \cup S_{r}}\left\{\lambda_{k}\right\} u_{S_{i} \cup S_{j} \cup \cdots \cup S_{r}}=\sum_{i=1}^{r} \lambda_{S_{i}} u_{S_{i}}-\sum_{i=1}^{r-1} \sum_{j=1}^{r} \lambda_{S_{i}} u_{S_{i} \cup S_{j}} \\
+\sum_{i=1}^{r-2} \sum_{j=i+1}^{r-1} \sum_{l=j+1}^{r} \lambda_{S_{i}} u_{S_{i} \cup S_{j} \cup S_{l}}+\cdots+(-1)^{r-1} \lambda_{S_{i}} u_{S_{i} \cup S_{j} \cup \cdots \cup S_{r},}
\end{gathered}
$$

the second equality holding by the definition of the modified game, and the third equality holding as $\lambda_{S_{1}} \leq \lambda_{S_{2}} \leq \ldots \leq \lambda_{S_{r}}$. On the other hand,

$$
u_{S_{1}}^{\lambda} \vee u_{S_{2}}^{\lambda} \vee \cdots \vee u_{S_{r}}^{\lambda}=\lambda_{S_{1}} u_{S_{1}} \vee \lambda_{S_{2}} u_{S_{2}} \vee \cdots \vee \lambda_{S_{r}} u_{S_{r}} .
$$

Let us prove that both expression (3) and (4) coincide. For $S \subseteq N$ with $S_{1} \nsubseteq \subseteq S$, $S_{2} \nsubseteq S, \ldots, S_{r} \nsubseteq S$, using (3), $\left(u_{S_{1}} \vee u_{S_{2}} \vee \cdots \vee u_{S_{r}}\right)^{\lambda}(S)=0$ and using $(4), u_{S_{1}}^{\lambda} \vee u_{S_{2}}^{\lambda} \vee \cdots \vee$ $u_{S_{r}}^{\lambda}=\max \left\{\lambda_{S_{1}} u_{S_{1}}(S), \ldots, \lambda_{S_{r}} u_{S_{r}}(S)\right\}=0$. Thus, both expressions (3) and (4) coincide for these coalitions.

Then, suppose that $S \subseteq N$ is such that $\left\{S_{i_{1}}, \ldots, S_{i_{t}}\right\}$ is the subset of those elements in $\left\{S_{1}, \ldots, S_{r}\right\}$ such that $S_{i} \subseteq S, j=1, \ldots, t$. Let us assume again, without loss of generality, that $\lambda_{S_{i_{1}}} \leq \cdots \leq \lambda_{S_{i_{t}}}$. Then, using (3),

$$
\begin{aligned}
& \left(u_{S_{1}} \vee u_{S_{2}} \vee \cdots \vee u_{S_{r}}\right)^{\lambda}(S)=\sum_{j=1}^{t} \lambda_{S_{i_{j}}} u_{S_{i_{j}}}(S)-\sum_{j=1}^{t-1} \sum_{l=j+1}^{t} \lambda_{S_{i_{j}}} u_{S_{i_{j}} \cup S_{i_{l}}}(S) \\
& +\cdots+(-1)^{t-1} \lambda_{S_{i_{1}}} u_{S_{i_{1}} \cup \cdots \cup S_{i_{t}}}(S)=\sum_{j=1}^{t} \lambda_{S_{i_{j}}}-\sum_{j=1}^{t-1} \sum_{l=j+1}^{t} \lambda_{S_{i_{j}}}+\cdots+(-1)^{t-1} \lambda_{S_{i_{1}}} \\
& =\lambda_{S_{i_{1}}}\left[1-\left(\begin{array}{c}
t-1 \\
1
\end{array}\right)+\left(\begin{array}{c}
t-1 \\
2
\end{array}\right)+\cdots+(-1)^{t-1}\left(\begin{array}{c}
t-1 \\
t-1
\end{array}\right)\right] \\
& +\lambda_{S_{t_{2}}}\left[1-\left(\begin{array}{c}
t-2 \\
1
\end{array}\right)+\cdots+(-1)^{t-2}\left(\begin{array}{c}
t-2 \\
t-2
\end{array}\right)\right] \\
& +\ldots+\lambda_{S_{i_{t}}}=\lambda_{S_{i_{t}}}
\end{aligned}
$$

and using (4)

$$
\begin{gathered}
\left(\lambda_{S_{1}} u_{S_{1}} \vee \lambda_{S_{2}} u_{S_{2}} \vee\right. \\
\left.=\max \left\{\lambda_{S_{i_{1}}}, \ldots, \lambda_{S_{i_{t}}}\right\}=\lambda_{S_{S_{i_{t}}}} u_{S_{r}}\right)(S)=\max \left\{\lambda_{S_{1}} u_{S_{1}}(S), \ldots, \lambda_{S_{t}} u_{S_{t}}(S)\right\} \\
\end{gathered}
$$

which completes the proof.

Proposition 7. Given $(N, v, \lambda),(N, w, \lambda) \in G_{p, \Lambda}^{N}$ we have that

$$
(v \vee w)^{\lambda}=v^{\lambda} \vee w^{\lambda}
$$

Proof. Suppose $\mathcal{M W C}(N, v)=\left\{S_{1}, \ldots, S_{r}\right\}$ and $\mathcal{M U C}(N, w)=\left\{T_{1}, \ldots, T_{m}\right\}$. Then $v=$ $u_{S_{1}} \vee \cdots \vee u_{S_{r}}$ and $w=u_{T_{1}} \vee \cdots \vee u_{T_{m}}$. Consequently,

$$
\begin{gathered}
(v \vee w)^{\lambda}=\left[\left(u_{S_{1}} \vee \cdots \vee u_{S_{r}}\right) \vee\left(u_{T_{1}} \vee \cdots \vee u_{T_{m}}\right)\right]^{\lambda} \\
=\left(u_{S_{1}} \vee \cdots \vee u_{S_{r}} \vee u_{T_{1}} \cdots \vee u_{T_{m}}\right)^{\lambda} \\
=u_{S_{1}}^{\lambda} \vee \cdots \vee u_{S_{r}}^{\lambda} \vee u_{T_{1}}^{\lambda} \vee \cdots \vee u_{T_{m}}^{\lambda}=\left(u_{S_{1}}^{\lambda} \vee \cdots \vee u_{S_{r}}^{\lambda}\right) \vee\left(u_{T_{1}}^{\lambda} \vee \cdots \vee u_{T_{m}}^{\lambda}\right)
\end{gathered}
$$




$$
=\left(u_{S_{1}} \vee \cdots \vee u_{S_{r}}\right)^{\lambda} \vee\left(u_{T_{1}} \vee \cdots \vee u_{T_{m}}\right)^{\lambda}=v^{\lambda} \vee w^{\lambda},
$$

the second and the fourth equality hold because $\vee$ is clearly associative, and the third equality holds because of Lemma 1.

In the following proposition, which needs two previous lemmas, we prove that the modified game of the minimum of the voting games is the minimum of its modified games.

Lemma 2. If $\left(N, u_{S_{1}}, \lambda\right),\left(N, u_{S_{2}}, \lambda\right), \ldots,\left(N, u_{S_{r}}, \lambda\right) \in G_{p, \Lambda}^{N}$, then,

$$
\left(u_{S_{1}} \wedge u_{S_{2}} \wedge \ldots \wedge u_{S_{r}}\right)^{\lambda}=u_{S_{1}}^{\lambda} \wedge u_{S_{2}}^{\lambda} \wedge \ldots \wedge u_{S_{r}}^{\lambda} .
$$

Proof. We have

$$
\left(u_{S_{1}} \wedge u_{S_{2}} \wedge \cdots \wedge u_{S_{r}}\right)^{\lambda}=\left(u_{S_{1} \cup S_{2} \cup \cdots \cup S_{r}}\right)^{\lambda}=\lambda_{S_{1} \cup \cdots \cup S_{r}} u_{S_{1} \cup \cdots \cup S_{r}} .
$$

If, as usual, we assume, without loss of generality, that $\lambda_{S_{1}} \leq \cdots \leq \lambda_{S_{r}}$, then $\lambda_{S_{1} \cup \cdots \cup S_{r}}=$ $\min _{k \in S_{1} \cup \cdots \cup S_{r}}\left\{\lambda_{k}\right\}=\lambda_{S_{1}}$, and thus

$$
\left(u_{S_{1} \cup \cdots \cup S_{r}}\right)^{\lambda}=\lambda_{S_{1}} u_{S_{1} \cup \cdots \cup S_{r}} .
$$

On the other hand,

$$
u_{S_{1}}^{\lambda} \wedge u_{S_{2}}^{\lambda} \wedge \cdots \wedge u_{S_{r}}^{\lambda}=\lambda_{S_{1}} u_{S_{1}} \wedge \lambda_{S_{2}} u_{S_{2}} \wedge \lambda_{S_{r}} u_{S_{r}} .
$$

Let us prove that both expressions (5) and (6) coincide.

It is trivial that for $S \subseteq N$, such that there exists $k \in\{1, \ldots, r\}$ with $S_{k} \nsubseteq \subseteq S$

$$
\lambda_{S_{1}} u_{S_{1} \cup \cdots \cup S_{r}}(S)=0
$$

and

$$
\left(\lambda_{S_{1}} u_{S_{1}} \wedge \lambda_{S_{2}} u_{S_{2}} \wedge \cdots \wedge \lambda_{S_{r}} u_{S_{r}}\right)(S)=\min \left\{\lambda_{S_{1}} u_{S_{1}}(S), \ldots, \lambda_{S_{r}} u_{S_{r}}(S)\right\}=0 .
$$

Suppose then, $S \subseteq N$ is such that $S_{1} \subseteq S, \ldots, S_{r} \subseteq S$. Then

$$
\left(u_{S_{1}} \wedge u_{S_{2}} \wedge \cdots \wedge u_{S_{r}}\right)^{\lambda}=\lambda_{S_{1}} u_{S_{1} \cup \cdots \cup S_{r}}(S)=\lambda_{S_{1}}
$$

and

$$
\left(\lambda_{S_{1}} u_{S_{1}} \wedge \lambda_{S_{2}} u_{S_{2}} \wedge \lambda_{S_{r}} u_{S_{r}}\right)(S)=\min \left\{\lambda_{S_{1}}, \ldots, \lambda_{S_{r}}\right\}=\lambda_{S_{1}},
$$

which completes the proof.

Lemma 3. Given $(N, v),(N, w) \in G_{p}^{N}$, with $\mathcal{M W C}(N, v)=\left\{S_{1}, \ldots, S_{r}\right\}$ and $\mathcal{M W C}(N, w)=$ $\left\{T_{1}, \ldots, T_{m}\right\}$, it holds that

$$
v \wedge w=\left(u_{S_{1}} \wedge u_{T_{1}}\right) \vee\left(u_{S_{1}} \wedge u_{T_{2}}\right) \vee \cdots \vee\left(u_{S_{r}} \wedge u_{T_{m}}\right)=\vee_{i=1}^{r} \vee_{j=1}^{m}\left(u_{S_{i}} \wedge u_{T_{j}}\right) .
$$

Proof. Under the hypothesis $v=u_{S_{1}} \vee u_{S_{2}} \vee \cdots \vee u_{S_{r}}$ and $w=u_{T_{1}} \vee u_{T_{2}} \vee \cdots \vee u_{T_{m}}$, and thus $v \wedge w=\left(u_{S_{1}} \vee u_{S_{2}} \vee \cdots \vee u_{S_{r}}\right) \wedge\left(u_{T_{1}} \vee u_{T_{2}} \vee \cdots \vee u_{T_{m}}\right)$. If $S \subseteq N$,

$$
\begin{gathered}
(v \wedge w)(S)=\left[\left(u_{S_{1}} \vee u_{S_{2}} \vee \cdots \vee u_{S_{r}}\right) \wedge\left(u_{T_{1}} \vee u_{T_{2}} \vee \cdots \vee u_{T_{m}}\right)\right](S) \\
=\min \left\{\left(u_{S_{1}} \vee u_{S_{2}} \vee \cdots \vee u_{S_{r}}\right)(S),\left(u_{T_{1}} \vee u_{T_{2}} \vee \cdots \vee u_{T_{m}}\right)(S)\right\} \\
=\min \left\{\max \left\{u_{S_{1}}(S), u_{S_{2}}(S), \ldots, u_{S_{r}}(S)\right\}, \max \left\{u_{T_{1}}(S), u_{T_{2}}(S), \ldots, u_{T_{m}}(S)\right\}\right\} \\
=\left\{\begin{array}{cc}
1 & \text { if there is } i \in\{1, \ldots, r\} \text { and } j \in\{1, \ldots, m\} \text { such that } S_{i} \subseteq S \text { and } T_{j} \subseteq S \\
0 & \text { otherwise. }
\end{array}\right.
\end{gathered}
$$


Then $v \wedge w=1-\prod_{i=1}^{r} \prod_{j=1}^{m}\left(1-u_{S_{i} \cup T_{j}}\right)$. $S \subseteq N$,

On the other hand, for $i \in\{1, \ldots, r\}, j \in\{1, \ldots, m\}\}, u_{S_{i}} \wedge u_{T_{j}}=u_{S_{i} \cup T_{j}}$, and thus, if

$$
\begin{gathered}
{\left[\left(u_{S_{1}} \wedge u_{T_{1}}\right) \vee\left(u_{S_{1}} \wedge u_{T_{2}}\right) \vee \cdots \vee\left(u_{S_{r}} \wedge u_{T_{m}}\right)\right](S)} \\
=\left(u_{S_{1} \cup T_{1}} \vee u_{S_{1} \cup T_{2}} \vee \cdots \vee u_{S_{r} \cup T_{m}}\right)(S)
\end{gathered}
$$$$
=\left\{\begin{array}{c}
1 \\
0 \quad \text { if there is } i \in\{1, \ldots, r\} \text { and } j \in\{1, \ldots, m\} \text { such that } S_{i} \cup T_{j} \subseteq S \\
\text { otherwise, }
\end{array}\right.
$$

which is equal to

$$
=1-\prod_{i=1}^{r} \prod_{j=1}^{m}\left(1-u_{S_{i} \cup T_{j}}\right),
$$

and then the proof is completed.

Proposition 8. Given $(N, v, \lambda),(N, w, \lambda) \in G_{p, \Lambda}^{N}$ we have that

$$
(v \wedge w)^{\lambda}=v^{\lambda} \wedge w^{\lambda}
$$

Proof. Suppose $\mathcal{M W C}(N, v)=\left\{S_{1}, \ldots, S_{r}\right\}$ and $\mathcal{M W C}(N, w)=\left\{T_{1}, \ldots, T_{m}\right\}$. Then $v=$ $u_{S_{1}} \vee \cdots \vee u_{S_{r}}$ and $w=u_{T_{1}} \vee \cdots \vee u_{T_{m}}$. Consequently,

$$
\begin{gathered}
(v \wedge w)^{\lambda}=\left[\left(u_{S_{1}} \vee \cdots \vee u_{S_{r}}\right) \wedge\left(u_{T_{1}} \vee \cdots \vee u_{T_{m}}\right)\right]^{\lambda} \\
=\left[\vee_{i=1}^{r} \vee_{j=1}^{m}\left(u_{S_{i}} \wedge u_{T_{j}}\right)\right]^{\lambda}=\left[\vee_{i=1}^{r} \vee_{j=1}^{m} u_{S_{i} \cup T_{j}}\right]^{\lambda} \\
=\vee_{i=1}^{r} \vee_{j=1}^{m} u_{S_{i} \cup T_{j}}^{\lambda}=\vee_{i=1}^{r} \vee_{j=1}^{m}\left(u_{S_{i}} \wedge u_{T_{j}}\right)^{\lambda} \\
=\vee_{i=1}^{r} \vee_{j=1}^{m}\left(u_{S_{i}}^{\lambda} \wedge u_{T_{j}}^{\lambda}\right)=\left(u_{S_{1}}^{\lambda} \vee \cdots \vee u_{S_{r}}^{\lambda}\right) \wedge\left(u_{T_{1}}^{\lambda} \vee \cdots \vee u_{T_{m}}^{\lambda}\right) \\
=\left(u_{S_{1}} \vee \cdots \vee u_{S_{r}}\right)^{\lambda} \wedge\left(u_{T_{1}} \vee \cdots \vee u_{T_{m}}\right)^{\lambda}=v^{\lambda} \wedge w^{\lambda},
\end{gathered}
$$

the second and the seventh equality hold because of Lemma 3, the fourth and the eighth equality using Lemma 1, and the sixth equality because of Lemma 2.

\section{The Banzhaf Value for Voting Games with Players Having Different Cooperation Abilities}

First, we define allocation rules in this setting.

Definition 4. An allocation rule on $G_{p, \Lambda}^{N}$ is a function $\psi: G_{p, \Lambda}^{N} \rightarrow \mathbb{R}^{n}$ that assigns to each $(N, v, \boldsymbol{\lambda}) \in G_{p, \Lambda^{\prime}}^{N} \psi(N, v, \boldsymbol{\lambda}) \in \mathbb{R}^{n}$, satisfying $\psi(N, v, \mathbf{0})=\mathbf{0}$. For $i=1, . ., n, \psi_{i}(N, v, \boldsymbol{\lambda})$ represents the outcome or payoff for Player i in $(N, v, \lambda)$.

Manuel and Martín [28] introduced (and characterized) the allocation rule for games with players having different bargaining abilities that assigns to every $(N, v, \lambda)$ the Shapley value of the modified game $\left(N, v^{\lambda}\right)$. In the following, we will define and study the allocation rule on $G_{p, \Lambda}^{N}$ that assigns to each $(N, v, \lambda)$ the Banzhaf value of $\left(N, v^{\lambda}\right)$.

Definition 5. The allocation rule $\beta$ on $G_{p, \Lambda}^{N}$ is defined for all $(N, v, \lambda) \in G_{p, \Lambda}^{N}$ as $\beta(N, v, \lambda)=$ $B\left(N, v^{\lambda}\right)$.

Remark 6. The restriction of $\beta$ to the family $\{(N, v, \mathbf{1})\}_{(N, v) \in G_{p}^{N}} \subsetneq G_{p, \Lambda}^{N}$ coincides with the Banzhaf value on $G_{p}^{N}$. Moreover, for all $(N, v, \lambda)$ with $\lambda_{i}=\lambda$ for all $i \in N, \beta(N, v, \lambda)=$ $\lambda . B(N, v)$. 
For voting games with players having different bargaining abilities, the defined value can be calculated as the linear combination of Banzhaf values of appropriate (classical) voting games. The proof of this result is straightforward from Proposition 5 , and then it is omitted.

Proposition 9. Given $(N, v, \lambda) \in G_{p, \Lambda}^{N}$, suppose $\operatorname{MWC}(N, v)=\left\{S_{1}, \ldots, S_{r}\right\}$ is the family of the minimal winning coalitions of $(N, v)$. If $\lambda_{S_{i}}=\min _{j \in S_{i}}\left\{\lambda_{j}\right\}$ for $j=1,2, \ldots, r$, with $\lambda_{S_{1}} \leq \lambda_{S_{2}} \leq$ $\cdots \leq \lambda_{S_{r}}$, and $\lambda_{S_{0}}=0$, then,

$$
\beta(N, v, \lambda)=\sum_{h=0}^{r-1}\left(\lambda_{S_{h+1}}-\lambda_{S_{h}}\right) \cdot B\left(N, v_{\left.\right|_{S_{h+1} \cup \cdots \cup S_{r}}}\right) .
$$

In the next proposition we prove that for convex voting games with players having different bargaining abilities, its Banzhaf value is upper-bounded by the Banzhaf value in the original voting game. Hence, by reducing the cooperation ability, the power is also reduced.

Proposition 10. Let $(N, v, \lambda) \in G_{p, \Lambda}^{N}$, with $(N, v)$ a convex game. Then

$$
\beta_{i}(N, v, \lambda) \leq B_{i}(N, v), \text { for all } i \in N
$$

Proof. If $(N, v)$ is null, the result is straightforward. Otherwise, $v=u_{S}$ for some $S \subseteq N$. Then, $\beta_{i}(N, v, \lambda)=\frac{\min _{k \in S}\left\{\lambda_{k}\right\}}{2^{s-1}}$ for $i \in S$ and zero in another case. As $B_{i}(N, v)=\frac{1}{2^{s-1}}$ for $i \in S$ and zero otherwise, the result is proved.

Nevertheless, in superadditive voting games with players having different cooperation abilities, a particular player can increase his Banzhaf value with respect to the one obtained in the original game, as it is shown in the next example.

Example 4. Consider $(N, v, \lambda)$ with $N=\{1,2,3\}, v=u_{\{1,2\}}+u_{\{1,3\}}-u_{\{1,2,3\}}, \lambda=$ $(1,0.9,1)$. Then, $B(N, v)=\left(\frac{3}{4}, \frac{1}{4}, \frac{1}{4}\right)$, and $\beta(N, v, \lambda)=\left(\frac{29}{40}, \frac{9}{40}, \frac{11}{40}\right)$.

The following proposition, whose proof is straightforward from (iv) of Proposition 6, states that the defined Banzhaf value for games with players having different bargaining abilities is monotonous in the weights.

Proposition 11. Let $(N, v, \lambda),\left(N, v, \lambda^{\prime}\right) \in G_{p, \Lambda}^{N}$ with $(N, v)$ a superadditive game. If $\lambda_{i}^{\prime} \geq \lambda_{i}$, and $\lambda_{j}^{\prime}=\lambda_{j}$ for $j \neq i$, then

$$
\beta_{i}\left(N, v, \lambda^{\prime}\right) \geq \beta_{i}(N, v, \lambda) .
$$

As mentioned, Radzik et al. [27] introduced a weighted Banzhaf value, $\beta^{R, w}$, that (unlike our proposal) respects the total power but is not monotonous in the weights. According to this linear value $\beta^{R, w}$, in a game $\left(N, u_{S}\right)$ with players' weights $w=\left(w_{1}, \ldots, w_{n}\right) \in\left(\mathbb{R}^{+}\right)^{n}$, each player $i \in N$ receives

$$
\beta^{R, w}\left(N, u_{S}\right)=\left\{\begin{array}{cl}
\sum_{i \in S}^{w_{i}} w_{i} \cdot \frac{1}{2^{s-1}}, & \text { if } i \in S \\
0, & \text { if } i \notin S .
\end{array}\right.
$$


Example 5. Consider the voting game $(N, v) \in G_{p}^{N}$ with $N=\{1,2,3\}$ and

$$
v(S)=\left\{\begin{array}{cc}
1, & \text { if } S=\{1,2\}, S=\{1,3\} \text { or } S=\{1,2,3\} \\
0, & \text { otherwise. }
\end{array}\right.
$$

In terms of the unanimity basis, $v$ can be written as:

$$
v=u_{\{1,2\}}+u_{\{1,3\}}-u_{\{1,2,3\}} .
$$

Suppose that the vector of weights is $w=(0.01,0.3,0.4)$. Then,

$$
\beta^{R, w}(N, v)=(0.0248,0.378,0.346) .
$$

The counterintuitive behavior of $\beta^{R, w}$ can be seen in this case, since Player 2 who is symmetrical with 3 in the game, and has less weight, gets a higher payoff.

On the other hand, if Player 2's weight decreases from 0.3 to 0.2 , keeping the rest of the weights equal, the value in this case is $\beta^{R, w}(N, v)=(0.0319,0.394,0.323)$. It is observed that Player 2, with a smaller weight than before, has greater power.

\section{Characterizations of the Defined Value}

First we introduce some properties that allow us to characterize the defined value.

Definition 6. An allocation rule $\psi: G_{p, \Lambda}^{N} \rightarrow \mathbb{R}^{n}$ satisfies the $\lambda$-total power if

$$
\sum_{i \in N} \psi_{i}(N, v, \lambda)=\sum_{i \in N} \sum_{S \subseteq N \backslash\{i\}} \frac{1}{2^{n-1}}\left[v^{\lambda}(S \cup\{i\})-v^{\lambda}(S)\right] .
$$

In the following, we will denote

$$
\sum_{i \in N} \sum_{S \subseteq N \backslash\{i\}} \frac{1}{2^{n-1}}\left[v^{\lambda}(S \cup\{i\})-v^{\lambda}(S)\right]=T P(N, v, \lambda) .
$$

Proposition 12. The allocation rule on $G_{p, \Lambda}^{N}, \beta$, satisfies the $\lambda$-total power.

Proof. By the definition of $\beta$, we have

$$
\begin{aligned}
\sum_{i \in N} \beta_{i}(N, v \lambda)=B_{i}\left(N, v^{\lambda}\right) & =\sum_{i \in N} \sum_{S \subseteq N \backslash\{i\}} \frac{1}{2^{n-1}}\left[v^{\lambda}(S \cup\{i\})-v^{\lambda}(S)\right] \\
& =T P(N, v, \lambda) .
\end{aligned}
$$

In the following proposition, we obtain an alternative expression for $\operatorname{TP}(N, v, \lambda)$ when $(N, v, \lambda) \in G_{p, \Lambda}^{N}$.

Proposition 13. Let $(N, v, \lambda) \in G_{p, \Lambda}^{N}$. Suppose $\mathcal{M W C}(N, v)=\left\{S_{1}, \ldots, S_{r}\right\}, \lambda_{S_{i}}=\min _{k \in S_{i}}\left\{\lambda_{k}\right\}$ and $\lambda_{S_{1}} \leq \lambda_{S_{2}} \leq \cdots \leq \lambda_{S_{r}}$. Then we have

$$
\begin{gathered}
T P(N, v, \lambda)=\sum_{j=1}^{r} \frac{\lambda_{S_{j}}\left|S_{j}\right|}{2^{\left|S_{j}\right|-1}}-\sum_{j=1}^{r-1} \sum_{k=j+1}^{r} \frac{\lambda_{S_{j}}\left|S_{j} \cup S_{k}\right|}{2^{\left|S_{j} \cup S_{k}\right|-1}} \\
+\sum_{j=1}^{r-2} \sum_{k=j+1}^{r-1} \sum_{l=k+1}^{r} \frac{\lambda_{S_{j}}\left|S_{j} \cup S_{k} \cup S_{l}\right|}{2^{\left|S_{j} \cup S_{k} \cup S_{l}\right|-1}}+\cdots+(-1)^{r-1} \frac{\lambda_{S_{1}}\left|S_{1} \cup S_{2} \cup \cdots \cup S_{r}\right|}{2^{\left|S_{1} \cup S_{2} \cup \cdots \cup S_{r}\right|-1}}
\end{gathered}
$$


Proof. As for $(N, v, \lambda) \in G_{p, \Lambda^{\prime}}^{N}$

$$
\begin{aligned}
v^{\lambda}=\sum_{i=1}^{r} \lambda_{S_{i}} u_{S_{i}}- & \sum_{i=1}^{r-1} \sum_{j=i+1}^{r} \lambda_{S_{i}} u_{S_{i} \cup S_{j}}+\sum_{i=1}^{r-2} \sum_{j=i+1}^{r-1} \sum_{k=j+1}^{r} \lambda_{S_{i}} u_{S_{i} \cup S_{j} \cup S_{k}} \\
& +\cdots+(-1)^{r-1} \lambda_{S_{1}} u_{S_{1} \cup \cdots \cup S_{r}}
\end{aligned}
$$

we have

$$
\begin{gathered}
T P(N, v, \lambda)=\sum_{i \in N} \beta_{i}(N, v, \lambda)=\sum_{i \in N} B_{i}\left(N, v^{\lambda}\right) \\
=\sum_{j=1}^{r} \sum_{i \in S_{j}} \frac{\lambda_{S_{j}}}{2^{\left|S_{j}\right|-1}}-\sum_{j=1}^{r-1} \sum_{k=j+1}^{r} \sum_{i \in S_{j} \cup S_{k}} \frac{\lambda_{S_{j}}}{2^{\left|S_{j} \cup S_{k}\right|-1}} \\
+\sum_{j=1}^{r-2} \sum_{k=j+1}^{r-1} \sum_{l=k+1}^{r} \sum_{i \in S_{j} \cup S_{k} \cup S_{l}} \frac{\lambda_{S_{j}}}{2^{\left|S_{j} \cup S_{k} \cup S_{l}\right|-1}} \\
+\cdots+(-1)^{r-1} \sum_{i \in S_{1} \cup S_{2} \cup \cdots \cup S_{r}} \frac{\lambda_{S_{1}}}{2^{\left|S_{1} \cup S_{2} \cup \cdots \cup S_{r}\right|-1}} \\
=\sum_{j=1}^{r} \frac{\lambda_{S_{j}}\left|S_{j}\right|}{2^{\left|S_{j}\right|-1}}-\sum_{j=1}^{r-1} \sum_{k=j+1}^{r} \frac{\lambda_{S_{j}}\left|S_{j} \cup S_{k}\right|}{2^{\left|S_{j} \cup S_{k}\right|-1}}+\sum_{j=1}^{r-2} \sum_{k=j+1}^{r-1} \sum_{l=k+1}^{r} \frac{\lambda_{S_{j}}\left|S_{j} \cup S_{k} \cup S_{l}\right|}{2^{\left|S_{j} \cup S_{k} \cup S_{l}\right|-1}} \\
+\cdots+(-1)^{r-1} \frac{\lambda_{S_{1}}\left|S_{1} \cup S_{2} \cup \cdots \cup S_{r}\right|}{2^{\left|S_{1} \cup S_{2} \cup \cdots \cup S_{r}\right|-1}},
\end{gathered}
$$

which completes the proof.

Van den Brink and Gilles [40] defined necessary player in a TU game $(N, v)$ as a player $i \in N$ such that $v(S)=0$ if $i \notin S$.

Definition 7. An allocation rule $\psi: G_{p, \Lambda}^{N} \rightarrow \mathbb{R}^{n}$ satisfies equal treatment of necessary players if $\psi_{i}(N, v, \lambda)=\psi_{j}(N, v, \lambda)$ for $i, j$ necessary players in $(N, v)$.

Definition 8. An allocation rule $\psi$ defined on $G_{p, \Lambda}^{N}$ satisfies the null player property if, given $(N, v, \lambda) \in G_{p, \Lambda}^{N}$ and $i$ a null player in $(N, v)$, then $\psi_{i}(N, v, \lambda)=0$.

Definition 9. Ref. [8] An allocation rule $\psi$ defined on $G_{p, \Lambda}^{N}$ satisfies the transfer property if, for $(N, v, \lambda),(N, w, \lambda) \in G_{p, \Lambda^{\prime}}^{N}$

$$
\psi(N, v \vee w, \lambda)+\psi(N, v \wedge w, \lambda)=\psi(N, v, \lambda)+\psi(N, w, \lambda)
$$

Definition 10. Ref. [28] An allocation rule $\psi$ defined on $G_{p, \Lambda}^{N}$ satisfies the balanced contributions property if, for all $(N, v, \lambda) \in G_{p, \Lambda}^{N}$ and all $i, j \in N$,

$$
\psi_{i}(N, v, \lambda)-\psi_{i}\left(N, v, \lambda^{-j}\right)=\psi_{j}(N, v, \lambda)-\psi_{j}\left(N, v, \lambda^{-i}\right),
$$

$\lambda^{-k}$ being the vector $\left\{\lambda_{l}^{-k}\right\}_{l \in N} \in[0,1]^{n}$ with $\lambda_{l}^{-k}=\left\{\begin{array}{cc}\lambda_{l}, & \text { if } l \neq k \\ 0, & \text { if } l=k\end{array}\right.$

Definition 11. Ref. [41] An allocation rule $\psi$ defined on $G_{p, \Lambda}^{N}$ satisfies strong monotonicity if, given $(N, v, \lambda),(N, w, \lambda) \in G_{p, \Lambda}^{N}$ and $i \in N$ such that $v(S \cup\{i\})-v(S) \geq w(S \cup\{i\})-w(S)$ for all $S \subseteq N \backslash\{i\}$, then $\psi_{i}(N, v, \lambda) \geq \psi_{i}(N, w, \lambda)$. Strong monotonicity implies marginality [42], the property obtained when in the previous definition both inequalities are replaced by equalities. 
In the following proposition we prove that the defined rule, $\beta$, satisfies the properties defined above.

Proposition 14. The allocation rule $\beta: G_{p, \Lambda}^{N} \rightarrow \mathbb{R}^{n}$ satisfies the transfer and the null player properties, equal treatment of necessary players, balanced contributions, strong monotonicity and marginality.

Proof. (i) To prove that $\beta$ satisfies the transfer property, consider $(N, v, \lambda),(N, w, \lambda) \in$ $G_{p, \Lambda}^{N}$. Then, we have

$$
\begin{gathered}
\beta(N, v \vee w, \lambda)=B\left(N,(v \vee w)^{\lambda}\right)=B\left(N, v^{\lambda} \vee w^{\lambda}\right)=B\left(N, v^{\lambda}\right)+B\left(N, w^{\lambda}\right) \\
-B\left(N, v^{\lambda} \wedge w^{\lambda}\right)=\beta(N, v, \lambda)+\beta(N, w, \lambda)-\beta(N, v \wedge w, \lambda),
\end{gathered}
$$

the first and the last equality hold because of the definition of $\beta$, the second equality by the Proposition 7, and the third equality as the Banzhaf value defined on $G^{N}$ satisfies the transfer property.

(ii) Suppose that $(N, v, \lambda) \in G_{p, \Lambda}^{N}$ and $i \in N$ is a null player in $(N, v)$. Then, he is also a null player in $\left(N, v^{\lambda}\right)$. As the Banzhaf value on $G^{N}$ satisfies the null player property, the result is proved.

(iii) Suppose that $(N, v, \lambda) \in G_{p, \Lambda}^{N}$ and $i, j \in N$ are necessary players in $(N, v)$. Then, they are also necessary players in $\left(N, v^{\lambda}\right)$. As the Banzhaf value on $G^{N}$ satisfies equal treatment of necessary players (see [20]), the result is proved.

(iv) To prove that $\beta$ satisfies balanced contributions, let $(N, v, \lambda) \in G_{p, \Lambda}^{N}$. Suppose $\mathcal{M W C}(N, v)=\left\{S_{1}, \ldots, S_{r}\right\}$. For $i, j \in N$, we define

$$
\delta_{i j}(T)=\left\{\begin{array}{lc}
1, & \text { if } i, j \in T \\
0, & \text { otherwise }
\end{array}\right.
$$

Then,

$$
\begin{aligned}
& \beta_{i}(N, v, \lambda)-\beta_{i}\left(N, v, \lambda^{-j}\right)=B_{i}\left(N, v^{\lambda}\right)-B_{i}\left(N, v^{\lambda^{-j}}\right) \\
& =B_{i}\left(\sum_{k=1}^{r} \min _{t \in S_{k}}\left\{\lambda_{t}\right\} u_{S_{k}}-\sum_{k=1}^{r-1} \sum_{l=k+1}^{r} \min _{t \in S_{k} \cup S_{l}}\left\{\lambda_{t}\right\} u_{S_{k} \cup S_{l}}\right. \\
& \left.+\ldots+(-1)^{r-1} \min _{t \in S_{1} \cup \ldots \cup S_{r}}\left\{\lambda_{t}\right\} u_{S_{1} \cup \ldots \cup S_{r}}\right) \\
& -B_{i}\left(\sum_{k=1}^{r} \min _{t \in S_{k}}\left\{\lambda_{t}^{-j}\right\} u_{S_{k}}-\sum_{k=1}^{r-1} \sum_{l=k+1}^{r} \min _{t \in S_{k} \cup S_{l}}\left\{\lambda_{t}^{-j}\right\} u_{S_{k} \cup S_{l}}\right. \\
& \left.+\ldots+(-1)^{r-1} \min _{t \in S_{1} \cup \ldots \cup S_{r}}\left\{\lambda_{t}^{-j}\right\} u_{S_{1} \cup \ldots \cup S_{r}}\right) \\
& =\sum_{k=1}^{r} \min _{t \in S_{k}}\left\{\lambda_{t}\right\} \frac{\delta_{i j}\left(S_{k}\right)}{2^{\left|S_{k}\right|-1}}-\sum_{k=1}^{r-1} \sum_{l=k+1}^{r} \min _{t \in S_{k} \cup S_{l}}\left\{\lambda_{t}\right\} \frac{\delta_{i j}\left(S_{k} \cup S_{l}\right)}{2^{\left|S_{k} \cup S_{l}\right|-1}} \\
& +\ldots+(-1)^{r-1} \min _{t \in S_{1} \cup \ldots \cup S_{r}}\left\{\lambda_{t}\right\} \frac{\delta_{i j}\left(S_{1} \cup \ldots \cup S_{r}\right)}{2^{\left|S_{1} \cup \ldots \cup S_{r}\right|-1}},
\end{aligned}
$$

an expression which is symmetric in $i$ and $j$ and thus, $\beta_{i}(N, v, \lambda)-\beta_{i}\left(N, v, \lambda^{-j}\right)=$ $\beta_{j}(N, v, \lambda)-\beta_{j}\left(N, v, \lambda^{-i}\right)$.

(v) In [28] (p. 18) it is proved that if the marginal contributions of Player $i$ in $(N, v)$ are greater or equal than in $(N, w)$, then they are also greater or equal in $\left(N, v^{\lambda}\right)$ than in $\left(N, w^{\lambda}\right)$. Thus, the marginality of the Banzhaf value is inherited by $\beta$. 
Remark 7. We can observe that $\beta$ satisfies a generalized version of the balanced contributions property: given $(N, v, \lambda) \in G_{p, \Lambda}^{N}$ with $\mathcal{M W C}(N, v)=\left\{S_{1}, \ldots, S_{r}\right\}$, and $i, j \in N$

$$
\begin{aligned}
& \beta_{i}(N, v, \lambda)-\beta_{i}\left(N, v, \lambda^{-j, c}\right)=\beta_{j}(N, v, \lambda)-\beta_{j}\left(N, v, \lambda^{-i, c}\right), \\
& \text { with } \lambda^{-k, c}=\left\{\lambda_{l}^{-k, c}\right\}_{l \in N}=\left\{\begin{array}{cc}
\lambda_{l}, & \text { if } l \neq k \\
c<\lambda_{k}, & \text { if } l=k
\end{array} \quad k=i, j .\right. \\
& \text { This is so as } \left.\beta_{i}(N, v, \lambda)-\beta_{i}\left(N, v, \lambda^{-j, c}\right)=\sum_{k=1}^{r} \frac{\delta_{i j}\left(S_{k}\right)}{2^{\left|S_{k}\right|-1}}\left[\min _{t \in S_{k}}\left\{\lambda_{t}\right\}-\min \left\{\min _{t \in S_{k}}\left\{\lambda_{t}\right\}\right\}, c\right\}\right]- \\
& \sum_{k=1}^{r-1} \sum_{l=k+1}^{r} \frac{\delta_{i j}\left(S_{k} \cup S_{l}\right)}{2^{\left|S_{k} \cup S_{l}\right|-1}}\left[\min _{t \in S_{k} \cup S_{l}}\left\{\lambda_{t}\right\}-\min \left\{\min _{t \in S_{k} \cup S_{l}}\left\{\lambda_{t}\right\}, c\right\}\right] \\
& +\ldots+(-1)^{r-1} \frac{\delta_{i j}\left(S_{1} \cup \ldots \cup S_{r}\right)}{2^{\left|S_{1} \cup \ldots \cup S_{r}\right|-1}}\left[\min _{t \in S_{1} \cup \ldots \cup S_{r}}\left\{\lambda_{t}\right\}-\min \left\{\min _{t \in S_{1} \cup \ldots \cup S_{r}}\left\{\lambda_{t}\right\}, c\right\}\right] \text {, an expression which }
\end{aligned}
$$
is symmetric in $i$, and $j$.

Then we provide three characterizations of $\beta$. The first one is parallel to the one of Dubey and Shapley [8].

Proposition 15. $\beta$ is the unique allocation rule on $G_{p, \Lambda}^{N}$ satisfying $\lambda$-total power, the null player property, equal treatment of necessary players, and the transfer property.

Proof. It is already proved that $\beta$ defined on $G_{p, \Lambda}^{N}$ satisfies these four properties. Reciprocally, let $\psi: G_{p, \Lambda}^{N} \rightarrow \mathbb{R}^{n}$ be an allocation rule satisfying $\lambda$-total power, the null player property, equal treatment of necessary players and the transfer property. We will prove that $\psi(N, v, \lambda)=\beta(N, v, \lambda)$ for all $(N, v, \lambda) \in G_{p, \Lambda}^{N}$ by induction on $r$, the number of minimal winning coalitions of $(N, v)$. If $r=1$, and $\mathcal{M L C C}(N, v)=\left\{S_{1}\right\}, v=u_{S_{1}}$ and thus all $i \in S_{1}$ is a necessary player in $(N, v)$ and all $i \notin S_{1}$ is a null player. Then, for all $i \notin S_{1}$, using the null player property, $\psi_{i}\left(N, u_{S_{1}}, \lambda\right)=0$ and also $\beta_{i}\left(N, u_{S_{1}}, \lambda\right)=0$. Using the equal treatment of necessary players, $\psi_{i}(N, v, \lambda)=\psi_{j}(N, v, \lambda)$ for all $i, j \in S_{1}$. By the $\lambda$-total power property $\sum_{i \in S_{1}} \psi_{i}\left(N, u_{S_{1}}, \lambda\right)=\frac{\lambda_{S_{1}}\left|S_{1}\right|}{2^{\left|S_{1}\right|-1}}$ and thus, for $i \in S_{1}, \psi_{i}\left(N, u_{S_{1}}, \lambda\right)=\frac{\lambda_{S_{1}}}{2^{\left|S_{1}\right|-1}}$ which coincides with $\beta_{i}\left(N, u_{S_{1}}, \lambda\right)$.

By the induction hypothesis, let us suppose that $\psi$ coincides with $\beta$ for $(N, v, \lambda)$ if the number of minimal winning coalitions of $(N, v)$ is $k \leq r$, and consider $(N, v, \lambda)$ with $\mathcal{M W C}(N, v)=\left\{S_{1}, \ldots, S_{r+1}\right\}$. Then $v=u_{S_{1}} \vee u_{S_{2}} \vee \cdots \vee u_{S_{r+1}}$. As $\psi$ satisfies the transfer property,

$$
\begin{gathered}
\psi(N, v, \lambda)=\psi\left(N, u_{S_{1}} \vee u_{S_{2}} \vee \cdots \vee u_{S_{r+1}}, \lambda\right)=\psi\left(N,\left(u_{S_{1}} \vee u_{S_{2}} \vee \cdots \vee u_{S_{r}}\right) \vee u_{S_{r+1}}, \lambda\right) \\
=\psi\left(N,\left(u_{S_{1}} \vee u_{S_{2}} \vee \cdots \vee u_{S_{r}}\right), \lambda\right)+\psi\left(N, u_{S_{r+1}}, \lambda\right) \\
-\psi\left(N,\left(u_{S_{1}} \vee u_{S_{2}} \vee \cdots \vee u_{S_{r}}\right) \wedge u_{S_{r+1}}, \lambda\right) .
\end{gathered}
$$

Since $\wedge$ is distributive with respect to $\vee$ (see Lemma 3), we have

$$
\begin{aligned}
\left(u_{S_{1}} \vee u_{S_{2}} \vee \cdots \vee u_{S_{r}}\right) & \wedge u_{S_{r+1}}=\left(u_{S_{1}} \wedge u_{S_{r+1}}\right) \vee\left(u_{S_{2}} \wedge u_{S_{r+1}}\right) \vee \cdots \vee\left(u_{S_{r}} \wedge u_{S_{r+1}}\right) \\
= & u_{S_{1} \cup S_{r+1}} \vee u_{S_{2} \cup S_{r+1}} \vee \cdots \vee u_{S_{r} \cup S_{r+1}}
\end{aligned}
$$

the last equality holds, as for all $\left(N, u_{S}\right),\left(N, u_{T}\right) \in G^{N}, u_{S} \wedge u_{T}=u_{S \cup T}$. Then,

$$
\begin{gathered}
\psi(N, v, \lambda)=\psi\left(N,\left(u_{S_{1}} \vee u_{S_{2}} \vee \cdots \vee u_{S_{r}}\right), \lambda\right) \\
+\psi\left(N, u_{S_{r+1}}, \lambda\right)-\psi\left(N, u_{S_{1} \cup S_{r+1}} \vee u_{S_{2} \cup S_{r+1}} \vee \cdots \vee u_{S_{r} \cup S_{r+1}}, \lambda\right),
\end{gathered}
$$


which, using the induction hypothesis, coincides with

$$
\begin{gathered}
\beta\left(N,\left(u_{S_{1}} \vee u_{S_{2}} \vee \cdots \vee u_{S_{r}}\right), \lambda\right) \\
+\beta\left(N, u_{S_{r+1}}, \lambda\right)-\beta\left(N, u_{S_{1} \cup S_{r+1}} \vee u_{S_{2} \cup S_{r+1}} \vee \cdots \vee u_{S_{r} \cup S_{r+1}}, \lambda\right) .
\end{gathered}
$$

Using again the distributive property of $\wedge$ with respect to $\vee$ and that $\beta$ satisfies the transfer property, this last expression is equal to

$$
\beta\left(N, u_{S_{1}} \vee u_{S_{2}} \vee \cdots \vee u_{S_{r+1}}, \lambda\right)=\beta(N, v, \lambda),
$$

which completes the proof.

Proposition 16. $\beta$ is the unique allocation rule on $G_{p, \Lambda}^{N}$ satisfying the $\lambda$-total power and balanced contributions.

Proof. It has been previously proved that $\beta$ satisfies $\lambda$-total power and balanced contributions. Reciprocally, suppose $\psi$ is an allocation rule on $G_{p, \Lambda}^{N}$ satisfying these two properties. We will prove that $\psi(N, v, \lambda)=\beta(N, v, \lambda)$ for all $(N, v, \lambda) \in G_{p, \Lambda}^{N}$ by induction on $\delta(\lambda)$, the cardinality of $d(\lambda)=\left\{\lambda_{i}, i \in N \mid \lambda_{i}>0\right\}$.

If $\delta(\lambda)=0$, then $\boldsymbol{\lambda}=\mathbf{0}$, and by the definition of the allocation rule, $\psi_{i}(N, v, \mathbf{0})=0=$ $\beta_{i}(N, v, \mathbf{0})$ for all $i \in N$.

Suppose $\delta(\lambda)=1$, and let $i \in N$ be such that $\lambda_{i}>0$. Then, as $\psi$ satisfies balanced contributions, for each $j \neq i, j \in N$,

$$
\psi_{i}(N, v, \lambda)-\psi_{i}\left(N, v, \lambda^{-j}\right)=\psi_{j}(N, v, \lambda)-\psi_{j}\left(N, v, \lambda^{-i}\right) .
$$

As $\lambda^{-j}=\lambda, \psi_{i}(N, v, \lambda)-\psi_{i}\left(N, v, \lambda^{-j}\right)=0$, and then $\psi_{j}(N, v, \lambda)=\psi_{j}\left(N, v, \lambda^{-i}\right)=$ $\psi_{j}(N, v, \mathbf{0})=0$. Since for $(N, v, \lambda)$ with only one cooperative player the $\lambda$-total power is zero, we have $\psi_{i}(N, v, \lambda)=\operatorname{TP}(N, v, \lambda)=0$. Taking into account that $\beta$ also satisfies $\lambda$-total power and balanced contributions, we have the similarly $\beta_{i}(N, v, \lambda)=0$ for all $i \in N$, and thus both rules coincide.

Because of the induction hypothesis, let us assume that $\psi(N, v, \lambda)=\beta(N, v, \lambda)$ for all $(N, v) \in G_{p, \Lambda}^{N}$ and all $\lambda$ such that $\delta(\lambda) \leq r-1$, and consider $(N, v, \lambda)$ with $\delta(\lambda)=r$.

We can assume, without loss of generality, that $\lambda_{1}>0, \lambda_{2}>0, \ldots, \lambda_{r}>0$, and $\lambda_{r+1}=\lambda_{r+2}=\lambda_{n}=0$. Then, as $\psi$ satisfies balanced contributions, for $i \in\{1, \ldots, r\}$ and $j \in\{r+1, \ldots, n\}$, similarly as in the case of $\delta(\lambda)=1,0=\psi_{i}(N, v, \lambda)-\psi_{i}\left(N, v, \lambda^{-j}\right)=$ $\psi_{j}(N, v, \lambda)-\psi_{j}\left(N, v, \lambda^{-i}\right)$ (the first equality holding as $\lambda=\lambda^{-j}$ and the second as $\psi$ satisfies balanced contributions), which implies $\psi_{j}(N, v, \lambda)=\psi_{j}\left(N, v, \lambda^{-i}\right)=\beta_{j}\left(N, v, \lambda^{-i}\right)$, the last equality holding by the induction hypothesis. As $\beta$ also satisfies balanced contributions, $0=\beta_{i}(N, v, \lambda)-\beta_{i}\left(N, v, \lambda^{-j}\right)=\beta_{j}(N, v, \lambda)-\beta_{j}\left(N, v, \lambda^{-i}\right)$, and finally $\psi_{j}(N, v, \lambda)=$ $\beta_{j}(N, v, \lambda)$ for all $j \in\{r+1, \ldots, n\}$.

On the other hand, if $i, j \in\{1, \ldots, r\}$, by the balanced contributions property,

$$
\psi_{i}(N, v, \lambda)-\psi_{i}\left(N, v, \lambda^{-j}\right)=\psi_{j}(N, v, \lambda)-\psi_{j}\left(N, v, \lambda^{-i}\right),
$$

which implies

$$
\begin{aligned}
& \psi_{i}(N, v, \lambda)-\psi_{j}(N, v, \lambda)=\psi_{i}\left(N, v, \lambda^{-j}\right)-\psi_{j}\left(N, v, \lambda^{-i}\right) \\
= & \beta_{i}\left(N, v, \lambda^{-j}\right)-\beta_{j}\left(N, v, \lambda^{-i}\right)=\beta_{i}(N, v, \lambda)-\beta_{j}(N, v, \lambda),
\end{aligned}
$$

the second equality holding again by the induction hypothesis and the last one holding as $\beta$ also satisfies balanced contributions. Then, $\psi_{i}(N, v, \lambda)-\beta_{i}(N, v, \lambda)=\psi_{j}(N, v, \lambda)-$ 
$\beta_{j}(N, v, \lambda)$ for all $i, j \in\{1, \ldots, r\}$, and thus, $\psi_{i}(N, v, \lambda)-\beta_{i}(N, v, \lambda)=c$ for all $i \in$ $\{1, \ldots, r\}$. Using the $\lambda$-total power of both rules,

$$
\begin{gathered}
r c=\sum_{i=1}^{r}\left[\psi_{i}(N, v, \lambda)-\beta_{i}(N, v, \lambda)\right]=\sum_{i=1}^{r} \psi_{i}(N, v, \lambda)-\sum_{i=1}^{r} \beta_{i}(N, v, \lambda) \\
=T P(N, v, \lambda)-\sum_{i=r+1}^{n} \psi_{i}(N, v, \lambda)-\left[T P(N, v, \lambda)-\sum_{i=r+1}^{n} \beta_{i}(N, v, \lambda)\right]=0,
\end{gathered}
$$

and thus $c=0$, which completes the proof.

The following characterization can be considered parallel to that provided by Nowak [12].

Proposition 17. $\beta$ is the unique allocation rule in $G_{p, \Lambda}^{N}$ satisfying $\lambda$-total power, the equal treatment of necessary players, and marginality. As marginality is a weaker property than strong monotonicity, and $\beta$ satisfies strong monotonicity, the result also holds when replacing marginality by strong monotonicity.

Proof. It has been previously proved that $\beta$ satisfies $\lambda$-total power, the equal treatment of necessary players' property, and marginality. Reciprocally, suppose $\psi$ is an allocation rule on $G_{p, \Lambda}^{N}$ satisfying these three axioms. We will prove that $\psi=\beta$ by induction on $r$, the number of minimal winning coalitions of $(N, v)$.

If $r=1$, then, there exists $S \subseteq N$ such that $v=u_{S}$. All $i \in S$ are necessary players in $(N, v)$, and thus $\psi_{i}(N, v, \lambda)=c$ for all $i \in S$. On the other hand, for $i \notin S$, if $w=\mathbf{0}$, $v(T \cup\{i\})-v(T)=0=w(T \cup\{i\})-w(T)$ for all $T \subseteq N \backslash\{i\}$. Then, using the marginality property $\psi_{i}(N, v, \lambda)=\psi_{i}(N, \mathbf{0}, \boldsymbol{\lambda})$ for $i \notin S$. By the $\lambda$-total power $\psi_{i}(N, \mathbf{0}, \boldsymbol{\lambda})=0$ for all $i \in N$, and thus, $\psi_{i}(N, v, \lambda)=0=\beta_{i}(N, v, \lambda)$ for $i \notin S$. Then, using again the $\lambda$-total power, for $i \in S$

$$
\psi_{i}(N, v, \lambda)=c=\frac{T P(N, v, \lambda)}{|S|}=\frac{|S| \min _{j \in S}\left\{\lambda_{j}\right\}}{2^{s-1}|S|}=\frac{\min _{j \in S}\left\{\lambda_{j}\right\}}{2^{s-1}}=\beta_{i}(N, v, \lambda),
$$

and thus $\psi(N, v, \lambda)=\beta(N, v, \lambda)$ for all $i \in N$.

Suppose, then, by the induction hypothesis that the result is true for all $(N, v, \lambda)$ with $r \leq k-1$ and consider $(N, v, \lambda)$ with $r=k$ and $\mathcal{M W C}(N, v)=\left\{S_{1}, \ldots, S_{k}\right\}$. Then, $v=u_{S_{1}} \vee \cdots \vee u_{S_{k}}=\mathbf{1}-\prod_{i=1}^{r}\left(\mathbf{1}-u_{S_{i}}\right)$. If $i \in N$ is such that $i \notin \bigcap_{t=1}^{k} S_{t}$, let consider the game $\left(N, v_{i}\right)$ with $v_{i}=\bigvee_{S_{t}: i \in S_{t}} u_{S_{t}}$. We have, for all $S \subseteq N \backslash\{i\}$,

$$
v_{i}(S \cup\{i\})-v_{i}(S)=v_{i}(S \cup\{i\})=v(S \cup\{i\})-v(S),
$$

and thus, $\psi_{i}\left(N, v_{i}, \lambda\right)=\psi_{i}(N, v, \lambda)$ because of the marginality property.

By induction hypothesis, $\psi_{i}\left(N, v_{i}, \lambda\right)=\beta_{i}\left(N, v_{i}, \lambda\right)$ as the cardinality of $\mathcal{M W C}\left(N, v_{i}\right)$ is $\leq k-1$. Moreover, $\beta_{i}(N, v, \lambda)=B_{i}\left(N, v^{\lambda}\right)=B_{i}\left(N, v_{i}^{\lambda}\right)=\beta_{i}\left(N, v_{i}, \lambda\right)$; the second equality holding as $B_{i}\left(N, v^{\lambda}\right)-B_{i}\left(N, v_{i}^{\lambda}\right)=B_{i}\left(N,\left(v-v_{i}\right)^{\lambda}\right)=0$, because $i$ is a null player in $v-v_{i}$, and thus, in $\left(v-v_{i}\right)^{\lambda}$, and the Banzhaf value satisfies the null player property. Then, $\psi_{i}(N, v, \lambda)=\psi_{i}\left(N, v_{i}, \lambda\right)=\beta_{i}\left(N, v_{i}, \lambda\right)=\beta_{i}(N, v, \lambda)$.

On the other hand, if $i \in \bigcap_{t=1}^{k} S_{t}$, then $i$ is a necessary player in $(N, v)$. All necessary players have equal rewards, and thus using $\lambda$-total power, the payoff for all of them is uniquely determined. 


\section{Multilinear Extension of a TU Game with Players Having Different Bargaining Abilities and the Defined Value, $\beta$}

The multilinear extension of a TU game $(N, v) \in G^{N}$ was introduced by Owen [33] as a function $f:[0,1]^{n} \rightarrow \mathbb{R}$ given by

$$
f\left(x_{1}, \ldots, x_{n}\right)=\sum_{\varnothing \neq S \subseteq N}\left\{\prod_{i \in S} x_{i} \prod_{i \notin S}\left(1-x_{i}\right)\right\} v(S) .
$$

The multilinear extension can also be calculated in terms of the dividends of the game as

$$
f\left(x_{1}, \ldots, x_{n}\right)=\sum_{\varnothing \neq S \subseteq N} \Delta_{v}(S) \prod_{j \in S} x_{j} .
$$

It is a useful tool when calculating the Shapley value and the Banzhaf value. In particular, Owen [5,33] proved that for $i \in N$

$$
S h_{i}(N, v)=\int_{0}^{1}\left(\frac{\partial}{\partial x_{i}} f\right)(t, \ldots, t) d t,
$$

and

$$
B_{i}(N, v)=\frac{\partial}{\partial x_{i}} f\left(\frac{1}{2}, \ldots, \frac{1}{2}\right)
$$

Manuel and Martín [28] generalized the multilinear extensions to the setting of games with players having different bargaining abilities. For $(N, v, \lambda) \in G_{\Lambda}^{N}$, the multilinear extension is given by

$$
f_{\lambda}\left(x_{1}, \ldots, x_{n}\right)=\sum_{S \subseteq N, s \geq 2}\left[\Delta_{v}(S) \min _{i \in S}\left\{\lambda_{i}\right\} \prod_{i \in S} x_{i}\right]+\sum_{i=1}^{n} \Delta_{v}(\{i\}) x_{i}
$$

Particularizing this expression to a voting game with players having different bargaining abilities or different levels of cooperation $(N, v, \lambda)$, in which $\mathcal{M W C}(N, v)=$ $\left\{S_{i}, \ldots, S_{r}\right\}, \lambda_{S_{i}}=\min _{j \in S_{i}}\left\{\lambda_{j}\right\}$ with $\lambda_{S_{1}} \leq \cdots \leq \lambda_{S_{r}}$, we have

$$
\begin{gathered}
f_{\lambda}\left(x_{1}, \ldots, x_{n}\right)=\sum_{i=1}^{r} \lambda_{S_{i}} \prod_{l \in S_{i}} x_{l}-\sum_{i=1}^{r-1} \sum_{j=i+1}^{r} \lambda_{S_{i}} \prod_{l \in S_{i} \cup S_{j}} x_{l} \\
+\sum_{i=1}^{r-2} \sum_{j=i+1}^{r-1} \sum_{k=j+1}^{r} \lambda_{S_{i}} \prod_{l \in S_{i} \cup S_{j} \cup S_{k}} x_{l}+\cdots+(-1)^{r-1} \lambda_{S_{1}} \prod_{l \in S_{1} \cup \cdots \cup S_{r}} x_{l} .
\end{gathered}
$$

The following proposition gives an expression to calculate the Banzhaf value in $G_{p, \Lambda^{\prime}}^{N}$ $\beta$, in terms of the multilinear extensions.

Proposition 18. Given $(N, v, \lambda) \in G_{p, \Lambda}^{N}$ it holds that

$$
\beta_{i}(N, v, \lambda)=\frac{\partial}{\partial x_{i}} f_{\lambda}\left(\frac{1}{2}, \ldots, \frac{1}{2}\right) .
$$

Proof. Given $(N, v, \lambda) \in G_{p, \Lambda^{\prime}}^{N}$ suppose $\mathcal{M W C}(N, v)=\left\{S_{i}, \ldots, S_{r}\right\}, \lambda_{S_{i}}=\min _{k \in S_{i}}\left\{\lambda_{k}\right\}$ for $i=1, \ldots, r$ and, without loss of generality, $\lambda_{S_{1}} \leq \lambda_{S_{2}} \leq \ldots \leq \lambda_{S_{r}}$. Let us note, for $S \subseteq N$, $\delta_{S}(i)=\left\{\begin{array}{lc}1, & \text { if } i \in S \\ 0, & \text { otherwise. }\end{array}\right.$

Then, for $i=1, \ldots, n$

$$
\frac{\partial}{\partial x_{i}} f_{\lambda}\left(x_{1}, \ldots, x_{n}\right)=\sum_{j=1}^{r} \lambda_{S_{j}} \delta_{S_{j}}(i) \prod_{l \in S_{j}, l \neq i} x_{l}-\sum_{j=1}^{r-1} \sum_{k=j+1}^{r} \lambda_{S_{j}} \delta_{S_{j} \cup S_{k}}(i) \prod_{l \in S_{j} \cup S_{k}: l \neq i} x_{l}
$$




$$
+\cdots+(-1)^{r-1} \lambda_{S_{1}} \delta_{S_{1} \cup \cdots \cup S_{r}}(i) \prod_{l \in S_{1} \cup \cdots \cup S_{r}: l \neq i} x_{l} .
$$

Evaluating this partial derivative in $\left(\frac{1}{2}, \ldots, \frac{1}{2}\right)$ we obtain, for $i=1, \ldots, n$

$$
\begin{gathered}
\frac{\partial}{\partial x_{i}} f_{\lambda}\left(\frac{1}{2}, \ldots, \frac{1}{2}\right)=\sum_{j=1}^{r} \lambda_{S_{j}} \delta_{S_{j}}(i) \frac{1}{2^{\left|S_{j}\right|-1}}-\sum_{j=1}^{r-1} \sum_{k=j+1}^{r} \lambda_{S_{j}} \delta_{S_{j} \cup S_{k}}(i) \frac{1}{2^{\left|S_{j} \cup S_{k}\right|-1}} \\
+\cdots+(-1)^{r-1} \lambda_{S_{1}} \delta_{S_{1} \cup \cdots \cup S_{r}}(i) \frac{1}{2^{\left|S_{1} \cup \cdots \cup S_{r}\right|-1}}=\beta_{i}(N, v, \lambda)
\end{gathered}
$$

which completes the proof.

\section{Conclusions and Final Remarks}

In this paper, we have introduced an extension of the classical Banzhaf index of power for voting games to the setting in which players have different cooperation abilities. To do this, the original game is modified to a new one in which the imperfect cooperation reduces the dividend of each coalition, multiplying them by the minimum of members weights. The Banzhaf value of this modified game can be used to measure the power of players in these situations, generalizes the classical Banzhaf index of power, and admits several characterizations parallel to that existing in the literature and a new one.

The obtained results can be generalized in different ways. For example, by considering the same ideas, we can extend the introduced value to the space of all TU games, but also to the setting of TU games with players having different bargaining abilities and cooperation restricted by a graph [43] or by a priori coalitions. The framework can also be generalized assuming that the weights of players, instead of being fixed, can depend on the coalitions they are involved with, or on the other players. As mentioned, instead of assuming a given weight system, it is possible that a different approach may exist, in the spirit of [26]: to provide axioms that allow to characterize the proposed solution if, and only if a system of weights exists. This problem remains open and deserves attention.

Author Contributions: Conceptualization, C.M.M. and D.M.; methodology, C.M.M. and D.M.; software, C.M.M. and D.M.; validation, C.M.M. and D.M.; formal analysis, C.M.M. and D.M.; investigation, C.M.M. and D.M.; resources, C.M.M. and D.M.; data curation, C.M.M. and D.M.; writing-original draft preparation, C.M.M. and D.M.; writing-review and editing, C.M.M. and D.M.; visualization, C.M.M. and D.M.; supervision, C.M.M. and D.M.; project administration, C.M.M. and D.M.; funding acquisition, C.M.M. and D.M. All authors have read and agreed to the published version of the manuscript.

Funding: This research has been partially supported by the "Plan Nacional de I+D+i" of the Spanish Government under the project MTM2015-70550-P. D. Martín also wants to thank University Complutense of Madrid and Bank of Santander for his pre-doctoral contract. The authors wish to thank two anonymous referees and the editor for their useful suggestions and comments.

Institutional Review Board Statement: Not applicable.

Informed Consent Statement: Not applicable.

Data Availability Statement: Not applicable.

Conflicts of Interest: The authors declare no conflict of interest.

\section{References}

1. Shapley, L.S. A value for n-person games. In Annals of Mathematics Studies; Kuhn, H.W., Tucker, A.W., Eds.; Princeton Univ Press: Princeton, NJ, USA, 1953; pp. 307-317.

2. Penrose, L.S. The elementary statistics of majority voting. J. R. Stat. Soc. 1946, 109, 53-57. [CrossRef]

3. Banzhaf, J.F. Weighted voting doesn't work: A game theoretic approach. Rutgers Law Rev. 1965, 19, $317-343$.

4. Coleman, J.S. Control of Collectives and the Power of a Collectivity to Act. In Social Choice; Lieberman, B., Ed.; Gordon and Breach: London, UK, 1971; pp. 269-300.

5. Owen, G. Multilinear Extensions and the Banzhaf Value. Nav. Res. Logist. Q. 1975, 22, 741-750. [CrossRef] 
6. Owen, G. Evaluation of Presidential Election Game. Am. Political Sci. 1975, 69, 947-953. [CrossRef]

7. Roth, A. A note on values and multilinear extensions. Nav. Res. 1977, 24, 517-520. [CrossRef]

8. Dubey, P.; Shapley, L.S. Mathematical properties of the Banzhaf power index. Math. Oper. Res. 1979, 4, 99-131. [CrossRef]

9. Lehrer, E. An axiomatization of the Banzhaf value. Int. J. Game Theory 1988, 17, 89-99. [CrossRef]

10. Feltkamp, V. Alternative Axiomatic Characterizations of the Shapley and Banzhaf Values. Int. J. Game Theory 1995, 24, 179-186. [CrossRef]

11. Dragan, I. New mathematical properties of the Banzhaf value. Eur. J. Oper. Res. 1996, 95, 451-463. [CrossRef]

12. Nowak, A.S. On an axiomatization of the Banzhaf value without the additivity axiom. Int. J. Game Theory 1997, 26, 137-141. [CrossRef]

13. Laruelle, A.; Valenciano, F. Shapley-Shubik and Banzhaf indices revisited. Math. Oper. Res. 2001, 26, 89-104. [CrossRef]

14. Casajus, A. Marginality, differential marginality, and the Banzhaf value. Theory Decis. 2011, 71, 365-372. [CrossRef]

15. Owen, G. Modification of the Banzhaf-Coleman index for games with a priori unions. In Power, voting, and voting power. Physica, Heidelberg, Germany, 1981, pp. 232-238.

16. Alonso-Meijide, J.M.; Carreras, F.; Fiestras-Janeiro, M.G.; Owen, G. A comparative axiomatic characterization of the Banzhaf-Owen coalitional value. Decis. Support Syst. 2007, 43, 701-712. [CrossRef]

17. Lorenzo-Freire, S. New characterizations of the Owen and Banzhaf-Owen values using the intracoalitional balanced contributions property. Top 2017, 25, 579-600. [CrossRef]

18. Algaba, E.; Bilbao, J.M.; van den Brink, R.; Jiménez-Losada, A. An axiomatization of the Banzhaf value for cooperative games on antimatroids. Math. Methods Oper. Res. 2004, 59, 147-166. [CrossRef]

19. Van den Brink R. Banzhaf Permission Values for Games with a Permission Structure. In ICM Millennium Lectures on Games; Petrosyan L.A., Yeung, D.W.K., Eds.; Springer: Berlin/Heidelberg, Germany, 2003.

20. Van den Brink, R. Axiomatizations of Banzhaf permission values for games with a permission structure. Int. J. Game Theory 2010, 39, 445-466. [CrossRef]

21. Alonso-Meijide, J.M.; Fiestras-Janeiro, M.G. The Banzhaf value and communication situations. Nav. Res. Logist. (NRL) 2006, 53, 198-203. [CrossRef]

22. Gallego, I.; Fernández, J.R.; Jiménez-Losada, A.; Ordóñez, M. A Banzhaf value for games with fuzzy communication structure: Computing the power of the political groups in the European Parliament. Fuzzy Sets Syst. 2014, 255, 128-145. [CrossRef]

23. Tan, C.; Feng, W.; Han, W. On the Banzhaf-like Value for Cooperative Games with Interval Payoffs. Mathematics 2020, 8, 372. [CrossRef]

24. Fragnelli, V.; Pusillo, L. Multiobjective Games for Detecting Abnormally Expressed Genes. Mathematics 2020, 8, 350. [CrossRef]

25. Shapley, L.S. Additive and Nom-Additive Set Functions. Ph.D. Thesis, Princeton University, Princeton, NJ, USA, 1953.

26. Kalai, E.; Samet, D. On weigthed Shapley values. Int. J. Game Theory 1987, 16, 205-222. [CrossRef]

27. Radzik, T.; Nowak, A.S.; Driessen, T.S.H. Weighted Banzhaf values. Math. Methods. Oper. Res. 1997, 45, 109-118. [CrossRef]

28. Manuel, C.; Martín, D. A Monotonic Weighted Shapley Value. Group Decis. Negot. 2020, 29, 627-654. [CrossRef]

29. Aubin, J.P. Cooperative fuzzy games. Math. Oper. Res. 1981, 6, 1-13. [CrossRef]

30. Jiménez-Losada, A.; Fernández, J.R.; Ordóñez, M.; Grabisch, M. Games on fuzzy communication structures with Choquet players. Eur. J. Oper. Res. 2010, 207, 836-847. [CrossRef]

31. Jiménez-Losada, A.; Fernández, J.R.; Ordóñez, M. Myerson values for games with fuzzy communication structures. Fuzzy Sets Syst. 2013, 213, 74-90. [CrossRef]

32. Owen, G. A Note on the Shapley Value. Manag. Sci. 1968, 18, 731-732. [CrossRef]

33. Owen, G. Multilinear Extensions of Games. Manag. Sci. 1972, 18, 64-79. [CrossRef]

34. Harsanyi, J.C. A bargaining model for cooperative n-person games. In Contributions to the Theory of Games IV; Tucker, A.W., Luce, R.D., Eds.; Princeton Univ Press: Princeton, NJ, USA, 1959; pp. 325-355.

35. Dubey, P. On the uniqueness of the Shapley value. Int. J. Game Theory 1975, 4, 131-139. [CrossRef]

36. Choquet, G. Theory of capacities. Annales de I'institut Fourier 1953, 5, 131-295. [CrossRef]

37. Sugeno, M.; Murofushi, T. Choquet's integral as an integral form for a general class of fuzzy measures. Prepr. Second IFSA Congr. 1987, 1, 408-411.

38. Grabisch, M.; Murofushi, T.; Sugeno, M. Fuzzy measure of fuzzy events defined by fuzzy integrals. Fuzzy Sets Syst. 1992, 50, 293-313. [CrossRef]

39. Tsurumi, M.; Tanino, T.; Inuiguchi, M. A Shapley function on a class of cooperative fuzzy games. Eur. J. Oper. Res. 2001, 129, 596-618. [CrossRef]

40. Van den Brink, R.; Gilles, R.P. Axiomatizations of the conjunctive permission value for games with permission structures. Games Econ. Behav. 1996, 12, 113-126. [CrossRef]

41. Young, H.P. Monotonic solutions of cooperative games. Int. J. Game Theory 1985, 14, 65-72. [CrossRef]

42. Chun, Y. A new axiomatization of the Shapley value. Games Econ. Behav. 1989, 1, 119-130. [CrossRef]

43. Manuel, C.; Martín, D. A value for communication situations with players having different bargaining abilities. Ann. Oper. Res. 2020, 301, 1-22. 